\title{
A Note on an Analytic Solution for an Incompressible Fluid-Conveying Pipeline System
}

\author{
Vincent O. S. Olunloyo, ${ }^{1}$ Charles A. Osheku, ${ }^{2}$ and Patrick S. Olayiwola ${ }^{3}$ \\ ${ }^{1}$ Department of Systems Engineering, Faculty of Engineering, University of Lagos, Akoka-Yaba, Lagos 23401, Nigeria \\ ${ }^{2}$ Centre for Space Transport and Propulsion, National Space Research and Development Agency, \\ Federal Ministry of Science and Technology, FCT, PMB 437, Abuja, Nigeria \\ ${ }^{3}$ Department of Mechanical \& Biomedical Engineering, College of Engineering, Bells University of Technology, Ota 234037, \\ Ogun State, Nigeria \\ Correspondence should be addressed to Charles A. Osheku; charlesosheku2002@yahoo.com
}

Received 13 July 2016; Accepted 21 September 2016; Published 6 March 2017

Academic Editor: Mohammad Tawfik

Copyright (C) 2017 Vincent O. S. Olunloyo et al. This is an open access article distributed under the Creative Commons Attribution License, which permits unrestricted use, distribution, and reproduction in any medium, provided the original work is properly cited.

\begin{abstract}
This paper presents an integral transform analytic solution to the equations governing a fluid-conveying pipeline segment where a gyroscopic or Coriolis force effect is taken into consideration. The mathematical model idealizes a segment of the pipeline as an elastic beam conveying an incompressible fluid. It is clearly shown that when such a system is supported at both ends and in a free motion, the Coriolis force dissipates no energy (or simply does not work) as it generates conjugate complex vibratory components for all flow velocities. It is demonstrated that the modal natural frequencies can be computed from the algebraic products of the complex frequency pairs. Clearly, the patterns of the characteristics of the system's natural frequencies are seen partly when the real and imaginary components are plotted, as widely seen in the literature. Nonetheless, results from this study revealed that a continuity profile exists to connect the subcritical, critical, and postcritical vibratory behaviours when the absolute values are plotted for any velocity. In the meantime, the efficacy and versatility of this method against the usual assumed spatial or temporal modal solutions are demonstrated by confirming the predictions and validity of results of earlier workers such as Paidoussis, Ziegler, and others where pre- and postdivergence behaviours are exhibited.
\end{abstract}

\section{Introduction}

Fluid-conveying pipes are parts of the most common engineering examples of slender systems interacting with axial flows; another good example is the deployment of flexible conduits in the oil and gas exploration and production industry. A compendium of other examples can be found in Paidoussis [1] work spanning over the last 50 years. The list of examples is not limited to the field of engineering but cuts across other areas of human endeavor such as the study of pulmonary and urinary tract systems or even haemodynamics within human physiology.

These problems have generated a lot of research interests over the years partly because some served as models for studying the stability of certain classes of dynamical systems leading to the development of novel numerical and analytical methods for solving such problems. It has also turned out, over the years, that several of these techniques have found wider applications in other areas of research that otherwise appeared unrelated and have in fact occasionally led to the development of unanticipated practical applications and devices. Thus for linear dynamics for axial flows along slender structures, the pipe conveying fluid is regarded as the main paradigm. It also serves as model for classical problems involving axial momentum transport or axially moving continua such as high speed magnetic and paper tapes [1].

However, a careful study of the development of this area of research revealed that most of the research interests were curiosity-driven as some of the interesting phenomena observed occurred at velocities and conditions outside practical engineering and operational working limits as at 
that time [2]. This however gradually changed with the study of high velocity flow within light-gauge piping used in rocket engines and stability problems experienced by oil pipelines at modest conveyance speeds. Nowadays, people are looking at areas of direct applications, for example, in the behaviour of aspirating pipes for ocean mining and LNG in-situ production. This concept is to be utilized for the proposed offshore mining of methane liquid-crystal deposits and carbon sequestration. Here, the interest is in flowinduced vibrations and instabilities that can arise at high flow rates.

Furthermore, with the advent of High Pressure-High Temperature (HP-HT) oil and gas exploration and production, the lengths of the flexible risers deployed surely qualify them as hoses or pipe strings whose vibration behaviour is of interest in the exploration field.

In the modeling of the mechanics of fluid-conveying pipes, one of the terms that has received considerable attention over the years is the Coriolis force that was assigned the role of energy absorption that counters the centrifugal effect that normally arose in free motions. Broadly speaking, such an energy absorption affects the stability in conservative and nonconservative systems, as was shown by Section 7 of Elishakoff's work (2005) [2]; Öz and Boyaci (2000) [3]; Szmidt and Przybyłowicz (2013) [4]; Askarian et al. (2014) [5]; Kuiper and Metrikine (2004) [6]; Chellapilla and Simha (2008) [7]. Other contributors that attempted to investigate and explain the behaviour included Leklong et al. (2008) [8]; Al-Hilli and Ntayeesh (2013) [9]; Guo et al. (2006) [10]; Zhang et al. (2000) [11]; Modarres-Sadeghi and Païdoussis (2009) [12] as well as Ibrahim (2010) [13].

Principal among these findings is that while the centrifugal force imparts energy to the system, the Coriolis force absorbs energy from the system, such that the balance between the two, in the absence of dissipation, gives rise to flutter. However, when confronted with a nonconservative system the effect of the Coriolis force can lead to destabilization. These conclusions were arrived at partly on experimental work as can be found in [14, 15]. However, in the 1950s, proofs were claimed of researchers and mathematicians' findings showing that the Coriolis has negligible effects even on the molecular scale interaction.

Part of the interest in this present study rests on the fact that previous explanations in literature as to the effect of the Coriolis force on the natural frequency of the system derived from ad hoc heuristic arguments accompanied sometimes by authoritative and masterly analyses and interpretation of results of numerical and experiments. What is however missing is adequate proof based on the results of blind solutions of the unabridged governing differential equations for the linear problems as simple examples or justifications of such rationalizations.

A separate but issue related is that although the gyroscopic (Coriolis) forces do no work in the course of free motions, they nonetheless exert important influences on the overall dynamical behaviour of a pipeline system as pointed out in [1]. It would therefore be useful to know exactly what their influences are in such cases.
Another issue that has arisen over the years is that of the efficacy of the methodology. To be sure, several methods have been used to tackle the class of problems associated with the dynamics of fluid-conveying pipes but prominent amongst these is the original work of Gregory and Paidoussis and the sequel as presented in [1] where the use of an eigenfunction expansion in a modified Galerkin scheme was introduced. Part of the initial challenge was the absence of computers and the availability of validation modules to check the results of numerical work. With the development of the Finite element method more emphasis was placed on numerical schemes and laboratory experiments were framed up to confirm the predictions of these studies. Some of the other methods used for analyses over the years included the spectral method, for example, deployed by Lee and Park (2006) [16] or the differential transformation method recently applied by Qiao et al. (2006) [17].

Other recent works are those of Dodds Jr. and Runyan (1965) [18], who used flow visualization and velocity measurement to experimentally clarify the mechanism underlying the fluid-induced vibration in double T-junction of pipeline systems; Yamaguchi et al. (2016) [19], who used a solution method based on the Frobenius power series on a derived asymptotic model from the solutions of the PridmoreBrown equation for the Fourier transform of the vibrational fluid pressure; Kutin and Bajsić (2014) [20], who use smart materials; and Jweeg and Ntayeesh (2015) [21] who made use of application of method of multiple scales to analyse approximately the gyroscopic system for a nonlinear fluidconveying pipeline.

This paper further establishes the method of complex integral transforms (where the cosine and sine transforms are special cases) as one other effective method that can be used to tackle such problems within the context of linear theory for a start and is organized as follows. Section 1 introduces the problems under investigation. In Section 2, the analysis of the pipeline conveying an incompressible fluid with the governing partial differential equation and appropriate boundary conditions are presented. In Section 3, the complex and natural frequencies of the system are computed together with the relationship of the system's natural frequency with the flow critical velocity. Section 4 analyses the dynamic responses for purely elastic pipe in three cases, namely, simple supports at both ends, cantilever pipe, and a clamped-pinned ends pipe. In Section 5, results are analysed and discussed and Section 6 concludes the paper, whilst references are listed in the final section and at the end found the Appendices A and B.

\section{Analysis of the Pipeline System Conveying an Incompressible Fluid}

The homogeneous Partial Differential Equation (PDE) governing the flow-induced vibration of a pipeline conveying an incompressible fluid is given by

$$
\begin{aligned}
& \mathrm{EI} \frac{\partial^{4} w}{\partial x^{4}}+\left(\rho_{s}+\rho_{f}\right) \frac{\partial^{2} w}{\partial t^{2}}+\rho_{f} U^{2} \frac{\partial^{2} w}{\partial x^{2}}+2 \rho_{f} U \frac{\partial^{2} w}{\partial t \partial x} \\
& \quad=0
\end{aligned}
$$


where EI is the flexural rigidity of the pipe, $\rho_{s}$ and $\rho_{f}$ are the mass per unit length of pipe and fluid, respectively, flowing with a steady flow velocity $U$, and $w$ is the lateral deflection of the pipe. The parameters $x$ and $t$ are the axial coordinate and time variables, respectively.

In literature of fluid structure interaction mechanics, where fluid-induced vibrations are studied, (1) is often nondimensionalized as

$$
\frac{\partial^{4} \bar{W}}{\partial \xi^{4}}+\bar{U}^{2} \frac{\partial^{2} \bar{W}}{\partial \xi^{2}}+2 \sqrt{\beta} \bar{U} \frac{\partial^{2} \bar{W}}{\partial \xi \partial \tau}+\frac{\partial^{2} \bar{W}}{\partial \tau^{2}}=0,
$$

where

$$
\begin{aligned}
\bar{U} & =U L \sqrt{\frac{\rho_{f}}{\mathrm{EI}}}, \\
\beta & =\frac{\rho_{f}}{\rho_{s}+\rho_{f}}, \\
\tau & =\frac{t}{L^{2}} \sqrt{\frac{\mathrm{EI}}{\rho_{s}+\rho_{f}}}, \\
\xi & =\frac{x}{L}, \\
\bar{W} & =\frac{w}{L},
\end{aligned}
$$

whereas, for the present study, the dimensionless form is given by

$$
\frac{\partial^{4} \bar{W}}{\partial \bar{x}^{4}}+\zeta_{f} \bar{U}^{2} \frac{\partial^{2} \bar{W}}{\partial \bar{x}^{2}}+2 \zeta_{f} \bar{U} \frac{\partial^{2} \bar{W}}{\partial \bar{x} \partial \bar{t}}+\left(1+\zeta_{f}\right) \frac{\partial^{2} \bar{W}}{\partial \bar{t}^{2}}=0,
$$

where

$$
\begin{aligned}
\bar{U} & =U L \sqrt{\frac{\rho_{f}}{\mathrm{EI}}}, \\
\zeta_{f} & =\frac{\rho_{f}}{\rho_{s}}, \\
\bar{t} & =\frac{t}{\tau}, \\
\tau & =L^{2} \sqrt{\frac{\rho_{s}}{\mathrm{EI}},} \\
\bar{x} & =\frac{x}{L}, \\
\bar{W} & =\frac{w}{L},
\end{aligned}
$$

and the components involved are, respectively, the restoring, inertia, bending/centrifugal, and Coriolis force terms.

For comparison purposes, we present the following.

Case 1. We expect that if the pipeline segment is conveying a fluid and an eventual situation calls for the valve to be closed or the pump/compressor shut-off, an entrained fluid, be it hot, cold, pressurized, or otherwise, will be trapped in the pipe. When the flow velocity $\bar{U}=0$ (2) based on the nondimensionalizing method in the literature becomes

$$
\begin{aligned}
& \lim _{\bar{U} \rightarrow 0}\left(\frac{\partial^{4} \bar{W}}{\partial \xi^{4}}+\bar{U}^{2} \frac{\partial^{2} \bar{W}}{\partial \xi^{2}}+2 \sqrt{\beta_{f}} \bar{U} \frac{\partial^{2} \bar{W}}{\partial \xi \partial \tau}+\frac{\partial^{2} \bar{W}}{\partial \tau^{2}}\right) \\
& =\frac{\partial^{4} \bar{W}}{\partial \xi^{4}}+\frac{\partial^{2} \bar{W}}{\partial \tau^{2}}=0
\end{aligned}
$$

leading to

$$
\Omega_{n}= \pm \lambda^{2}= \pm n^{2} \pi^{2}
$$

which shows that there is no fluid inside the pipe if the flow velocity is zero. This is not to be so. However, considering (4), based on this paper's dimensionless method, with $\bar{U}=0$, it becomes

$$
\begin{aligned}
& \lim _{\bar{U} \rightarrow 0}\left(\frac{\partial^{4} \bar{W}}{\partial \bar{x}^{4}}+\zeta_{f} \bar{U}^{2} \frac{\partial^{2} \bar{W}}{\partial \bar{x}^{2}}+2 \zeta_{f} \bar{U} \frac{\partial^{2} \bar{W}}{\partial \bar{x} \partial \bar{t}}\right. \\
& \left.\quad+\left(1+\zeta_{f}\right) \frac{\partial^{2} \bar{W}}{\partial \bar{t}^{2}}\right)=\frac{\partial^{4} \bar{W}}{\partial \bar{x}^{4}}+\left(1+\zeta_{f}\right) \frac{\partial^{2} \bar{W}}{\partial \bar{t}^{2}}=0
\end{aligned}
$$

leading to

$$
\Omega_{n}= \pm \frac{\lambda^{2}}{\sqrt{\left(1+\zeta_{f}\right)}}= \pm \frac{n^{2} \pi^{2}}{\sqrt{\left(1+\zeta_{f}\right)}}
$$

Revealing that, the system's vibration, configuration, and response are strongly affected by the entrainment fluid in the pipe.

Case 2. If there is no fluid in the pipe, that is, $\zeta_{f}=\beta=0$, (2) and (4), respectively, become

$$
\begin{aligned}
& \lim _{\zeta_{f} \rightarrow 0}\left(\frac{\partial^{4} \bar{W}}{\partial \bar{x}^{4}}+\zeta_{f} \bar{U}^{2} \frac{\partial^{2} \bar{W}}{\partial \bar{x}^{2}}+2 \zeta_{f} \bar{U} \frac{\partial^{2} \bar{W}}{\partial \bar{x} \partial \bar{t}}\right. \\
& \left.+\left(1+\zeta_{f}\right) \frac{\partial^{2} \bar{W}}{\partial \bar{t}^{2}}\right)=0 \quad \text { that is } \frac{\partial^{4} \bar{W}}{\partial \bar{x}^{4}}+\frac{\partial^{2} \bar{W}}{\partial \bar{t}^{2}}=0
\end{aligned}
$$

leading to

$$
\Omega_{n}= \pm \lambda \sqrt{\lambda^{2}-\bar{U}^{2}}= \pm n \pi \sqrt{n^{2} \pi^{2}-\bar{U}^{2}}
$$




$$
\begin{aligned}
& \lim _{\zeta_{f} \rightarrow 0}\left(\frac{\partial^{4} \bar{W}}{\partial \bar{x}^{4}}+\zeta_{f} \bar{U}^{2} \frac{\partial^{2} \bar{W}}{\partial \bar{x}^{2}}+2 \zeta_{f} \bar{U} \frac{\partial^{2} \bar{W}}{\partial \bar{x} \partial \bar{t}}\right. \\
& \left.+\left(1+\zeta_{f}\right) \frac{\partial^{2} \bar{W}}{\partial \bar{t}^{2}}\right)=0
\end{aligned}
$$

$$
\text { that is } \frac{\partial^{4} \bar{W}}{\partial \bar{x}^{4}}+\frac{\partial^{2} \bar{W}}{\partial \bar{t}^{2}}=0 \text {. }
$$

The literature method (10) is showing that when no fluid is present in the pipe, there is still a flow velocity. This is not possible and is likely a fundamental error in physics. However, (12) shows that the restoring and the inertial accelerations in dimensionless form are the balancing vectors.

Case 3. We now examine the governing equation (1) in its original form without nondimensionalizing, such that, when $U=0$, it becomes

$$
\mathrm{EI} \frac{\partial^{4} w}{\partial x^{4}}+\left(\rho_{s}+\rho_{f}\right) \frac{\partial^{2} w}{\partial t^{2}}=0
$$

leading to

$$
\Omega_{n}= \pm \frac{\mathrm{EI}}{\rho_{s}\left(1+\zeta_{f}\right)}\left(\frac{\lambda}{L}\right)^{2}= \pm \frac{\mathrm{EI}}{\left(1+\zeta_{f}\right)} \frac{n^{2} \pi^{2}}{L^{2}} .
$$

In the absence of fluid in the pipe, that is, $m_{f}=0$.

$$
\mathrm{EI} \frac{\partial^{4} w}{\partial x^{4}}+\rho_{s} \frac{\partial^{2} w}{\partial t^{2}}=0
$$

leading to

$$
\Omega_{n}= \pm \frac{\mathrm{EI}}{\rho_{s}}\left(\frac{\lambda}{L}\right)^{2}= \pm \frac{\mathrm{EI}}{\rho_{s}} \frac{n^{2} \pi^{2}}{L^{2}} .
$$

It is confirmed that (13) is similar to (8), demonstrating that the natural frequency is dependent on the mass or density of fluid flowing in the pipeline.

Now, the following definitions hold for the Fourier complex integral transforms Wrede and Spiegel [22], Olayiwola [23], and Jeffrey [24]; namely,

$$
\begin{aligned}
F\{\bar{W}(\bar{x}, \bar{t})\} & =\frac{1}{\sqrt{2 \pi}} \int_{-\infty}^{\infty} e^{-i \lambda x} \bar{W}(\bar{x}, \bar{t}) d x \\
& =\bar{W}^{F}(\lambda, \bar{t}) ; \\
F^{-1}\left\{\bar{W}^{F}(\lambda, \bar{t})\right\} & =\frac{1}{\sqrt{2 \pi}} \int_{-\infty}^{\infty} e^{i \lambda x} \bar{W}^{F}(\lambda, \bar{t}) d \lambda \\
& =W(\bar{x}, \bar{t})
\end{aligned}
$$

such that in this case

$$
F(\bar{x}, \bar{t})= \begin{cases}0 & \text { when }-\infty \leq \bar{x}<0 \\ \bar{W}(\bar{x}, t) & \text { when } 0 \leq \bar{x} \leq 1 \\ 0 & \text { when } 1<\bar{x} \leq \infty\end{cases}
$$

Using (18) on (4), the following governing equation ensues in the transforms plane:

$$
\begin{aligned}
& \frac{d^{2} \bar{W}^{F}}{d \bar{t}^{2}}+i 2 \frac{\lambda \zeta_{f} \bar{U}}{\left(1+\zeta_{f}\right)} \frac{d \bar{W}^{F}}{d \bar{t}}+\frac{\left(\lambda^{4}-\zeta_{f} \bar{U}^{2} \lambda^{2}\right)}{\left(1+\zeta_{f}\right)} \bar{W}^{F} \\
& =\frac{1}{\left(1+\zeta_{f}\right)}\left(-\left.\bar{W}_{\overline{x x x}} e^{-i \lambda \bar{x}}\right|_{0} ^{1}-\left.i \lambda \bar{W}_{\overline{x x}} e^{-i \lambda \bar{x}}\right|_{0} ^{1}\right. \\
& \quad+\left.\left(\lambda^{2}-\zeta_{f} \bar{U}^{2}\right) \bar{W}_{\bar{x}} e^{-i \lambda \bar{x}}\right|_{0} ^{1} \\
& \left.\quad+\left.\left(i \lambda^{3}-\zeta_{f} \bar{U}^{2}\right) \bar{W} e^{-i \lambda \bar{x}}\right|_{0} ^{1}-\zeta_{f} \bar{U} \frac{d}{d \bar{t}}\left(\left.\bar{W} e^{-i \lambda \bar{x}}\right|_{0} ^{1}\right)\right),
\end{aligned}
$$

subject to simply supported conditions at both ends; namely,

$$
\begin{aligned}
& \bar{W}(0, t)=\bar{W}_{x x}(0, t)=0, \\
& \bar{W}(1, t)=\bar{W}_{x x}(1, t)=0 .
\end{aligned}
$$

In conjunction with the following conditions:

$$
\frac{d \bar{W}(0, t)}{d \bar{t}}=\frac{d \bar{W}(1, t)}{d \bar{t}}=0
$$

Substituting (20) into (19) leads to the following ordinary differential equation (ode) in the transform plane:

$$
\begin{gathered}
\left(1+\zeta_{f}\right) \frac{d^{2} \bar{W}^{F}}{d \bar{t}^{2}}+i 2 \lambda \zeta_{f} \bar{U} \frac{d \bar{W}^{F}}{d \bar{t}}+\left(\lambda^{4}-\zeta_{f} \bar{U}^{2} \lambda^{2}\right) \bar{W}^{F} \\
=\left(-\left.\bar{W}_{\overline{x x x}} e^{-i \lambda \bar{x}}\right|_{0} ^{1}+\left.\left(\lambda^{2}-\zeta_{f} \bar{U}^{2}\right) \bar{W}_{\bar{x}} e^{i \lambda \bar{x}}\right|_{0} ^{1}\right)=0 .
\end{gathered}
$$

This is a nonhomogeneous second-order ordinary differential equation in time domain. We can now proceed to solve for the frequency and displacement responses.

\section{Complex and Natural Frequencies}

In order to find the natural frequencies of the system we seek to solve the complimentary equation of the system in its Fourier complex transform plane by using the trial solution, namely,

$$
\bar{W}^{F}(\lambda, \bar{t})=A e^{s \bar{t}},
$$

to obtain the following characteristic equation:

$$
\left(\left(1+\zeta_{f}\right) s^{2}+i 2 \zeta_{f} \lambda \bar{U} s+\left(\lambda^{4}-\zeta_{f} \bar{U}^{2} \lambda^{2}\right)\right) A e^{s \bar{t}}=0 .
$$


The preceding equation can now be solved for the roots of $s$ to obtain complex conjugate pairs of the forms:

$$
\begin{aligned}
s_{1} & =\left(-i \frac{\zeta_{f} \bar{U} \lambda}{\left(1+\zeta_{f}\right)}\right. \\
& \left.+\sqrt{\left(i \frac{\zeta_{f} \bar{U} \lambda}{\left(1+\zeta_{f}\right)}\right)^{2}-\left(\frac{\left(\lambda^{4}-\zeta_{f} \bar{U}^{2} \lambda^{2}\right)}{\left(1+\zeta_{f}\right)}\right)}\right), \\
s_{2} & =\left(-i \frac{\zeta_{f} \bar{U} \lambda}{\left(1+\zeta_{f}\right)}\right) \\
& \left.-\sqrt{\left(i \frac{\zeta_{f} \bar{U} \lambda}{\left(1+\zeta_{f}\right)}\right)^{2}-\left(\frac{\left(\lambda^{4}-\zeta_{f} \bar{U}^{2} \lambda^{2}\right)}{\left(1+\zeta_{f}\right)}\right)}\right) .
\end{aligned}
$$

In order to isolate the effect of the Coriolis force, we introduce the expression

$$
\Omega_{\text {Cor. }}=i \frac{\zeta_{f} \bar{U} \lambda}{\left(1+\zeta_{f}\right)}
$$

into (25). On comparing these equations with the natural frequency, the relative frequencies are related as follows:

$$
\begin{aligned}
& \Omega_{1}=-\Omega_{n}\left(\xi_{n}+\sqrt{\left(1+\xi_{n}^{2}\right)}\right), \\
& \Omega_{2}=-\Omega_{n}\left(\xi_{n}-\sqrt{\left(1+\xi_{n}^{2}\right)}\right),
\end{aligned}
$$

where

$$
\xi_{n}=\frac{\Omega_{\text {cor }}}{\Omega_{n}} .
$$

Moreover, the product of the conjugate pairs gives the natural frequencies of the system; namely,

$$
\Omega_{n}^{2}=\Omega_{1} \times \Omega_{2}
$$

that is,

$$
\Omega_{n}^{2}=\left(\frac{\left(\lambda^{4}-\zeta_{f} \bar{U}^{2} \lambda^{2}\right)}{\left(1+\zeta_{f}\right)}\right)
$$

Although, from mathematical physics, the complex notation " $i$ " indicates that $\Omega_{\text {Cor. }}$ is acting perpendicularly to the natural frequency $\Omega_{n}$, nonetheless, an algebraic functional relation can be deduced as follows.

Substituting a square of (26) into (30) gives

$$
\Omega_{n}=\frac{\lambda^{2}}{\sqrt{\left(1+\zeta_{g}\right)}}\left(1-\frac{\Omega_{\text {cor }}^{2}\left(1+\zeta_{g}\right)^{2}}{\lambda^{4} \zeta_{g}}\right)^{1 / 2} .
$$

On expanding, it yields

$$
\begin{aligned}
\Omega_{n} & =\frac{\lambda^{2}}{\sqrt{\left(1+\zeta_{g}\right)}}\left\{1-\frac{\Omega_{\text {cor }}^{2}\left(1+\zeta_{g}\right)^{2}}{2 \lambda^{4} \zeta_{g}}\right. \\
& \left.+\frac{1}{2 !} \frac{\Omega_{\text {cor }}^{4}\left(1+\zeta_{g}\right)^{4}}{4 \lambda^{8} \zeta_{g}^{2}}-\frac{1}{3 !} \frac{3 \Omega_{\text {cor }}^{6}\left(1+\zeta_{g}\right)^{6}}{8 \lambda^{12} \zeta_{g}^{3}}+\cdots\right\}
\end{aligned}
$$

But for $\bar{U} \rightarrow 0, \Omega_{\text {cor }} \rightarrow 0$ or with $\Omega_{\text {cor }}\left(1+\zeta_{g}\right) \ll \lambda^{2} \sqrt{\zeta_{g}}$, tends to a limit; that is,

$$
\Omega_{n}=\frac{\lambda^{2}}{\sqrt{\left(1+\zeta_{g}\right)}} .
$$

The first observation to make from relations (18) and (22) is that the natural frequency is not in the same component with the frequency due to the Coriolis force.

Having noted this, it is also useful to conform with the general practice in the literature by expressing the result for the natural frequency, where possible, in a way that relates it to physical parameters or benchmarks associated with the flow. Thus from relation (19), it is straightforward to deduce the following.

(a) The critical axial velocity at which the natural frequency of the system is zero satisfies the relation

$$
\bar{U}_{\mathrm{cr}}^{2}=\frac{\lambda^{2}}{\zeta_{f}}
$$

and defines the condition for the onset of irregular oscillations.

(b) When there is no axial flow, that is, $\bar{U}=0$, the natural frequency of the system satisfies the relation

$$
\Omega_{0}^{2}=\left(\frac{\lambda^{4}}{\left(1+\zeta_{f}\right)}\right) .
$$

(c) The general relation for the magnitude of the natural frequency can be rearranged as

$$
\Omega_{n}^{2}=\Omega_{0}^{2}\left(1-\frac{\bar{U}^{2}}{\bar{U}_{\mathrm{cr}}^{2}}\right)
$$

This is a simple expression that relates the natural frequency to the critical flow velocity. Equation (34) actually proves the existence of such relationship as it could be argued that the results of the experiment of Dodds Jr. and Runyan (1965) [18] provide indirect evidence of the existence of such a relationship for the eigenfrequency mode $n=1$.

Furthermore, evaluation of the critical velocity and fundamental frequency can be carried out by substituting the appropriate eigenvalues into (24). Thus it can be asserted that, for this case, this method facilitates the derivation of explicit closed form expressions for possible design parameters such as the critical velocity. This method of solution also sets the stage for deriving equivalent results for $\bar{U}_{\mathrm{cr}}$ and $\Omega_{n}$ for the dynamic response when other effects such as damping for example are included. 


\section{Dynamic Response Analysis for Purely Elastic Pipe}

If we consider the dynamic response of the simple system of a purely elastic horizontal pipe with uniformly distributed loads of $f$ Newton per unit length, then (4) is transformed as follows:

$$
\frac{d^{2} \bar{W}^{F}}{d \bar{t}^{2}}+2 \Omega_{\mathrm{cor}} \frac{d \bar{W}^{F}}{d \bar{t}}+\Omega_{n}^{2} \bar{W}^{F}=\frac{J(\lambda)}{\left(1+\zeta_{f}\right)}
$$

where

$$
\begin{aligned}
\Omega_{\text {cor }} & =i \frac{\lambda \zeta_{f} \bar{U}}{\left(1+\zeta_{f}\right)}, \\
\Omega_{n}^{2} & =\frac{\left(\lambda^{4}-\zeta_{f} \bar{U}^{2} \lambda^{2}\right)}{\left(1+\zeta_{f}\right)}, \\
J(\lambda) & =\left(-\left.\bar{W}_{\overline{x x x}}(\bar{x}, 0) e^{-i \lambda \bar{x}}\right|_{0} ^{1}-\left.i \lambda \overline{W_{\overline{x x}}}(\bar{x}, 0) e^{-i \lambda \bar{x}}\right|_{0} ^{1}\right. \\
& +\left.\left(\lambda^{2}-\zeta_{f} \bar{U}^{2}\right) \bar{W} \overline{\bar{x}}(\bar{x}, 0) e^{-i \lambda \bar{x}}\right|_{0} ^{1} \\
& +\left.\left(i \lambda^{3}-\zeta_{f} \bar{U}^{2}\right) \bar{W}(\bar{x}, 0) e^{-i \lambda \bar{x}}\right|_{0} ^{1} \\
& \left.-\zeta_{f} \bar{U} \frac{d}{d \bar{t}}\left(\left.\bar{W}^{\prime}(\bar{x}, 0) e^{-i \lambda \bar{x}}\right|_{0} ^{1}\right)\right) .
\end{aligned}
$$

The general solution for the deflection response of the system is hence given as

$$
\bar{W}^{F}(\lambda, \bar{t})=A(\lambda) e^{-i \Omega_{1} \bar{t}}+B(\lambda) e^{-i \Omega_{2} \bar{t}}+\frac{J(\lambda)}{\Omega_{n}^{2}\left(1+\zeta_{f}\right)},
$$

where

$$
\begin{aligned}
A(\lambda)= & -\frac{1}{\Omega_{1}-\Omega_{2}}\left(\Omega_{1} H(\lambda, 0)-\frac{\Omega_{2} J(\lambda)}{\Omega_{n}^{2}\left(1+\zeta_{f}\right)}\right) \\
& -\frac{2 \Omega_{\text {cor }}}{\Omega_{1}-\Omega_{2}} H(\lambda, 0), \\
B(\lambda)= & \frac{2 \Omega_{\text {cor }}}{\Omega_{1}-\Omega_{2}} H(\lambda, 0) \\
& +\frac{1}{\Omega_{1}-\Omega_{2}}\left(\Omega_{2} H(\lambda, 0)-\frac{\Omega_{1} J(\lambda)}{\Omega_{n}^{2}\left(1+\zeta_{f}\right)}\right) .
\end{aligned}
$$

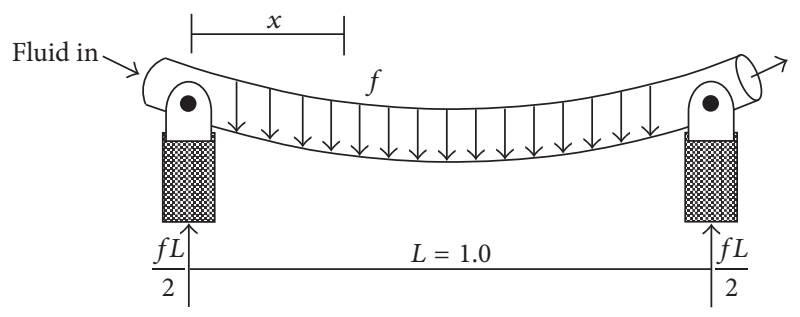

FIGURE 1: Simply supported beam with distributed load.

Therefore, in the Fourier plane, the dynamic response is obtained as

$$
\begin{aligned}
& \bar{W}^{F}(\lambda, \bar{t}) \\
& =-\frac{\Omega_{2} e^{-i \Omega_{2} \bar{t}}-\Omega_{1} e^{-i \Omega_{1} \bar{t}}-2 \Omega_{\mathrm{cor}}\left(e^{-i \Omega_{1} \bar{t}}-e^{-i \Omega_{2} \bar{t}}\right)}{\Omega_{1}-\Omega_{2}} \\
& \cdot H(\lambda, 0)-\frac{\left(\Omega_{1} e^{-i \Omega_{2} \bar{t}}-\Omega_{2} e^{-i \Omega_{1} \bar{t}}\right)-\left(\Omega_{1}-\Omega_{2}\right)}{\left(\Omega_{1}-\Omega_{2}\right)\left(1+\zeta_{f}\right)} \\
& \cdot \frac{J(\lambda)}{\Omega_{n}^{2}},
\end{aligned}
$$

where

$$
H(\lambda, 0)=\mathfrak{\Im}\{\bar{W}(\bar{x}, 0)\}
$$

We now examine the deflection responses for three cases of horizontal pipeline system with regard to solution (40) above.

Case 1.

Pipe with Simple Supports at Both Ends. In this case, as shown in Figure 1, the initial configuration of the pipe before it is dynamically excited shows that deflection is symmetric about the middle of the beam (or the pipe).

For this case, when the system is at the static state, time $\bar{t}=0$, the flow velocity is also zero, though the pipe might have trapped some fluid within it. (See Appendix for further analysis.)

The deflection function is given by Nash [25]:

$$
\bar{W}=\bar{w}\left(\frac{\bar{x}^{3}}{12}-\frac{\bar{x}^{4}}{24}-\frac{\bar{x}}{24}\right)
$$

where $\bar{w}=\beta_{f}\left(1+\zeta_{f}\right)$ and $\beta_{f}=m_{p} L / 2 E \widehat{I}$.

Now, (37) reduces to

$$
\begin{aligned}
& J_{s s}(\lambda)=\left(-\left.\bar{W}_{\overline{x x x}}(\bar{x}, 0) e^{-i \lambda \bar{x}}\right|_{0} ^{1}\right. \\
& \left.+\left.\left(\lambda^{2}-\zeta_{f} \bar{U}^{2}\right) \bar{W}_{\bar{x}}(\bar{x}, 0) e^{-i \lambda \bar{x}}\right|_{0} ^{1}\right)
\end{aligned}
$$


so that

$$
\begin{aligned}
& J_{s s}(\lambda)=\frac{\beta_{f}}{12}\left(1+\zeta_{f}\right)\left\{\left(\lambda^{2}-\zeta_{f} \bar{U}^{2}+12\right)\left(e^{-i \lambda}-1\right)\right\} \\
& H(\lambda, 0)=\Im\{\bar{W}(\bar{x}, 0)\} \\
& =\beta_{f}\left(1+\zeta_{f}\right) \int_{0}^{1}\left(\frac{\bar{x}^{3}}{12}-\frac{\bar{x}^{4}}{24}-\frac{\bar{x}}{24}\right) e^{-i \lambda \bar{x}} d \bar{x}
\end{aligned}
$$

The inverse Fourier transform is given as

$$
\bar{W}(\bar{x}, \bar{t})=\frac{1}{\sqrt{2 \pi}} \int_{-\infty}^{\infty} \bar{W}(\lambda, \bar{t}) e^{i \lambda \bar{x}} d \lambda
$$

$$
\begin{gathered}
\bar{W}(\bar{x}, \bar{t})=\left(-\int_{0}^{\infty} \frac{\Omega_{2} e^{-i \Omega_{2} \bar{t}}-\Omega_{1} e^{-i \Omega_{1} \bar{t}}-2 \Omega_{\mathrm{cor}}\left(e^{-i \Omega_{1} \bar{t}}-e^{-i \Omega_{2} \bar{t}}\right)}{\Omega_{1}-\Omega_{2}} H(\lambda, 0) e^{i \lambda \bar{x}} d \lambda\right. \\
\left.-\int_{0}^{\infty} \frac{\left(\Omega_{1}\left(e^{-i \Omega_{2} \bar{t}}-1\right)-\Omega_{2}\left(e^{-i \Omega_{1} \bar{t}}-1\right)\right)}{\left(\Omega_{1}-\Omega_{2}\right)} \frac{J_{s s}(\lambda)}{\Omega_{n}^{2}} e^{i \lambda \bar{x}} d \lambda\right) .
\end{gathered}
$$

That is,

$$
\begin{gathered}
\bar{W}(\bar{x}, \bar{t})=\bar{w}\left\{\left(\frac{\Omega_{2} e^{-i \Omega_{2} \bar{t}}-\Omega_{1} e^{-i \Omega_{1} \bar{t}}-2 \Omega_{\mathrm{cor}}\left(e^{-i \Omega_{1} \bar{t}}-e^{-i \Omega_{2} \bar{t}}\right)}{\Omega_{2}-\Omega_{1}}\right)\left(\frac{\bar{x}^{3}}{12}-\frac{\bar{x}^{4}}{24}-\frac{\bar{x}}{24}\right)\right. \\
\left.+\int_{0}^{\infty} \frac{\left(\Omega_{1}\left(e^{-i \Omega_{2} \bar{t}}-1\right)-\Omega_{2}\left(e^{-i \Omega_{1} \bar{t}}-1\right)\right)}{24\left(\Omega_{1}-\Omega_{2}\right)} \frac{\left(\lambda^{2}-\zeta_{f} \bar{U}^{2}+12\right)\left(e^{-i \lambda}-1\right)}{\lambda^{2}\left(\lambda^{2}-\zeta_{f} \bar{U}^{2}\right)} d \lambda\right\} .
\end{gathered}
$$

On enforcing the dynamic boundary conditions, namely, $\bar{W}(0, \bar{t})=\bar{W}(1, \bar{t})=0 ; \bar{W}_{\overline{x x}}(0, \bar{t})=\bar{W}_{\overline{x x}}(1, \bar{t})=0$, the system's dynamic response is given by

$$
\bar{W}(\bar{x}, \bar{t})
$$$$
=\bar{w}\left\{\left(\frac{\Omega_{2} e^{-i \Omega_{2} \bar{t}}-\Omega_{1} e^{-i \Omega_{1} \bar{t}}-2 \Omega_{\mathrm{cor}}\left(e^{-i \Omega_{1} \bar{t}}-e^{-i \Omega_{2} \bar{t}}\right)}{\Omega_{2}-\Omega_{1}}\right)\right.
$$$$
\cdot\left(\frac{\bar{x}^{3}}{12}-\frac{\bar{x}^{4}}{24}-\frac{\bar{x}}{24}\right)+i
$$$$
\frac{\left(\Omega_{1}\left(e^{-i \Omega_{2} \bar{t}}-1\right)-\Omega_{2}\left(e^{-i \Omega_{1} \bar{t}}-1\right)\right)}{2 n \pi\left(\Omega_{1}-\Omega_{2}\right)}\{\sin n \pi(1-\bar{x})
$$$$
-\sin n \pi \bar{x}\}\}
$$

or

$\bar{W}(\bar{x}, \bar{t})$

$$
=\bar{w}\left\{\left(\frac{\Omega_{2} e^{-i \Omega_{2} \bar{t}}-\Omega_{1} e^{-i \Omega_{1} \bar{t}}-2 \Omega_{\text {cor }}\left(e^{-i \Omega_{1} \bar{t}}-e^{-i \Omega_{2} \bar{t}}\right)}{\Omega_{2}-\Omega_{1}}\right)\right.
$$

$$
\begin{aligned}
& \cdot\left(\frac{\bar{x}^{3}}{12}-\frac{\bar{x}^{4}}{24}-\frac{\bar{x}}{24}\right)+i \\
& \cdot \frac{\left(\Omega_{1}\left(e^{-i \Omega_{2} \bar{t}}-1\right)-\Omega_{2}\left(e^{-i \Omega_{1} \bar{t}}-1\right)\right)}{2 n \pi\left(\Omega_{1}-\Omega_{2}\right)}\left(1-(-1)^{n+1}\right) \\
& \cdot \sin n \pi \bar{x}\}
\end{aligned}
$$

where

$$
\begin{aligned}
\Omega_{1} & =-\Omega_{\mathrm{cor}}-\sqrt{\Omega_{\mathrm{cor}}^{2}+\Omega_{n}^{2}}, \\
\Omega_{2} & =-\Omega_{\mathrm{cor}}+\sqrt{\Omega_{\mathrm{cor}}^{2}+\Omega_{n}^{2}}, \\
\Omega_{\mathrm{cor}} & =\frac{\left(\Omega_{2}+\Omega_{1}\right)}{i 2}=\frac{\zeta_{f} \bar{U} \lambda}{\left(1+\zeta_{f}\right)}, \\
\Omega_{n}^{2} & =\left(\frac{\left(\lambda^{4}-\zeta_{f} \bar{U}^{2} \lambda^{2}\right)}{\left(1+\zeta_{f}\right)}\right) \\
\lambda & =n \pi ; \\
\forall \lambda & =\bar{U}_{\mathrm{cr}}=\frac{n \pi}{\sqrt{\zeta_{f}}}(\text { See Appendix A for expanded analysis). }
\end{aligned}
$$


To enable us understand fully the characteristics of the natural frequencies of such a pipeline system, the three scenarios, namely, critical, subcritical, and postcritical flow points, are examined; that is, comparing (30) and (34), we deduce

$$
1-\Gamma^{2}=\frac{\lambda^{2}}{\Omega_{0}^{2}\left(1+\zeta_{f}\right)}\left(\lambda^{2}-\zeta_{f} \bar{U}^{2}\right),
$$

where

$$
\Gamma^{2}=\frac{\bar{U}^{2}}{\bar{U}_{\mathrm{cr}}^{2}} .
$$

The arguments for computing the residues in (40) are $\lambda=$ $\pm \bar{U} \sqrt{\zeta_{f}}$, when

$$
\frac{\lambda^{2}\left(\lambda^{2}-\zeta_{f} \bar{U}^{2}\right)}{\left(1+\zeta_{f}\right)}=0 .
$$

Nonetheless, a critical flow point $\Gamma=1$ is attained for any corresponding velocity, when (47) is zero; that is,

$$
\bar{U}=\bar{U}_{\mathrm{cr}} .
$$

For a subcritical flow $\Gamma<1$, this corresponds to the characteristics of the real part of natural frequency against flow velocity as normally seen in literature; that is,

$$
\bar{U}<\bar{U}_{\mathrm{cr}},
$$

and for any postcritical flow point, $\Gamma>1$ which corresponds to the characteristics of the imaginary part of natural frequency against flow velocity; that is,

$$
\bar{U}>\bar{U}_{\mathrm{cr}} .
$$

The point to note here is that, in actual practice, continuity must exist. As such the natural frequency cannot be zero perpetually for postcritical flow velocity. This necessitated the essence of the plot of absolute characteristics of the natural frequency for all regimes of flow, as demonstrated in this paper. These three scenarios are described graphically in Section 5

Case 2. A cantilever pipe is described.

From Figure 2, the initial configuration is described by

$$
\bar{W}(\bar{x}, 0)=\frac{\bar{w}}{24}\left(6 \bar{x}^{2}+4 \bar{x}^{3}-\bar{x}^{4}\right) ;
$$

thus,

$$
\begin{aligned}
& J_{c}(\lambda)=\left(\bar{W}_{\overline{x x x}}(0,0)-i \lambda \bar{W}_{\overline{x x}}(0,0)\right. \\
& +\left(\lambda^{2}-\zeta_{f} \bar{U}^{2}\right) \bar{W}_{\bar{x}}(1,0) e^{-i \lambda} \\
& \left.+\left(i \lambda^{3}-\zeta_{f} \bar{U}^{2}\right) \bar{W}(1,0) e^{-i \lambda}\right) .
\end{aligned}
$$

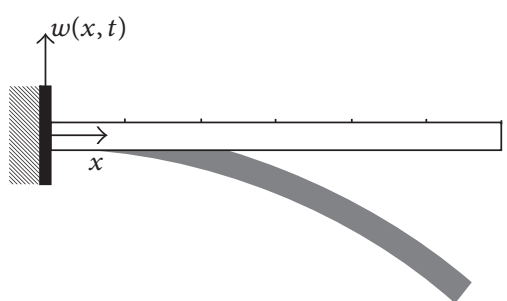

FIGURE 2: A fluid-conveying cantilever beam.

$$
\begin{aligned}
& J_{c}(\lambda) \\
& \quad=\bar{w}\left\{-12(2-i \lambda)+\left(4 \lambda^{2}+i \lambda^{3}-5 \bar{U}^{2} \zeta_{f}\right) e^{-i \lambda}\right\}, \\
& H(\lambda, 0)=\mathfrak{J}\{\bar{W}(\bar{x}, 0)\} \\
& \quad=\frac{\bar{w}}{24} \int_{0}^{1}\left(6 \bar{x}^{2}+4 \bar{x}^{3}-\bar{x}^{4}\right) e^{-i \lambda \bar{x}} d \bar{x} .
\end{aligned}
$$

The response for the fluid-conveying cantilever beam is therefore given as

$$
\begin{aligned}
& \bar{W}(\bar{x}, \bar{t}) \\
& =\bar{w}\left\{\frac{1}{24}\left(\frac{\Omega_{2} e^{-i \Omega_{2} \bar{t}}-\Omega_{1} e^{-i \Omega_{1} \bar{t}}-2 \Omega_{\mathrm{cor}}\left(e^{-i \Omega_{1} \bar{t}}-e^{-i \Omega_{2} \bar{t}}\right)}{\Omega_{2}-\Omega_{1}}\right)\right. \\
& \cdot\left(6 \bar{x}^{2}+4 \bar{x}^{3}-\bar{x}^{4}\right)-\frac{\Omega_{1}\left(e^{-i \Omega_{2} \bar{t}}-1\right)-\Omega_{2}\left(e^{-i \Omega_{1} \bar{t}}-1\right)}{\left(1+\zeta_{f}\right)\left(\Omega_{1}-\Omega_{2}\right)} \\
& \left.\quad \cdot \frac{J_{c}(\lambda) e^{i \lambda \bar{x}} d \lambda}{\Omega_{n}^{2}}\right\} ;
\end{aligned}
$$

that is,

$\bar{W}(\bar{x}, \bar{t})$

$$
\begin{aligned}
& =\frac{\bar{w}}{24}\left\{\left(\frac{\Omega_{2} e^{-i \Omega_{2} \bar{t}}-\Omega_{1} e^{-i \Omega_{1} \bar{t}}-2 \Omega_{\text {cor }}\left(e^{-i \Omega_{1} \bar{t}}-e^{-i \Omega_{2} \bar{t}}\right)}{\Omega_{2}-\Omega_{1}}\right)\right. \\
& \cdot\left(6 \bar{x}^{2}+4 \bar{x}^{3}-\bar{x}^{4}\right)-i 4 \\
& \cdot \frac{\Omega_{1}\left(e^{-i \Omega_{2} \bar{t}}-1\right)-\Omega_{2}\left(e^{-i \Omega_{1} \bar{t}}-1\right)}{2 \lambda\left(\Omega_{1}-\Omega_{2}\right)}(-24 \sin \lambda \bar{x} \\
& \left.\left.-\lambda^{2} \sin \lambda(1-\bar{x})+\lambda \cos \lambda \bar{x}+\lambda^{3} \cos \lambda(1-\bar{x})\right)\right\},
\end{aligned}
$$




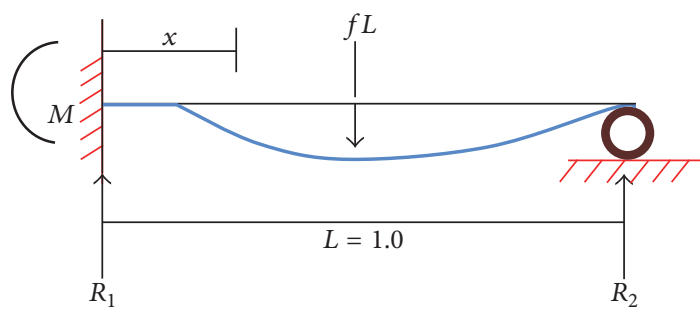

FIGURe 3: A clamped-pinned ended fluid-carrying pipe.

where $\bar{w}=\beta_{f}\left(1+\zeta_{f}\right)$

$$
\begin{aligned}
\Omega_{1} & =-\Omega_{\text {cor }}+\sqrt{\Omega_{\text {cor }}^{2}-\Omega_{n}^{2}}, \\
\Omega_{2} & =-\Omega_{\text {cor }}-\sqrt{\Omega_{\text {cor }}^{2}-\Omega_{n}^{2}}, \\
\Omega_{\text {cor }} & =i \frac{\bar{U} \zeta_{f} \lambda}{1+\zeta_{f}}, \\
\Omega_{n} & =\sqrt{\frac{\lambda^{4}-\lambda^{2} \bar{U}^{2} \zeta_{f}}{1+\zeta_{f}}},
\end{aligned}
$$

for which the characteristic equation at $\bar{x}=0,1$ is given by

$$
\left(2-\lambda^{2}\right) \tan \lambda-3 \lambda=0 .
$$

Case 3. For clamped and pinned ended supports: see Figure 3.

From Figure 4, the initial configuration is

$$
\bar{W}(\bar{x}, 0)=\frac{\bar{w}}{48} \bar{x}^{2}\left(-3+5 \bar{x}-2 \bar{x}^{2}\right) .
$$

Dynamic response of the clamped-pinned pipeline is given as

$$
\begin{aligned}
& \bar{W}(\bar{x}, \bar{t}) \\
& =\frac{\bar{w}}{48}\left\{\left(\frac{\Omega_{2} e^{-i \Omega_{2} \bar{t}}-\Omega_{1} e^{-i \Omega_{1} \bar{t}}-2 \Omega_{\mathrm{cor}}\left(e^{-i \Omega_{1} \bar{t}}-e^{-i \Omega_{2} \bar{t}}\right)}{\Omega_{2}-\Omega_{1}}\right)\right. \\
& \cdot \bar{x}^{2}\left(-3+5 \bar{x}-2 \bar{x}^{2}\right)-i \\
& \cdot \frac{1}{\lambda}\left(\frac{\Omega_{1}\left(e^{-i \Omega_{2} \bar{t}}-1\right)-\Omega_{2}\left(e^{-i \Omega_{1} \bar{t}}-1\right)}{\left(\Omega_{1}-\Omega_{2}\right)}\right) \\
& \cdot(6 \lambda \cos \lambda \bar{x}-30 \sin \lambda \bar{x}-18 \sin \lambda(1-\bar{x}))\},
\end{aligned}
$$

where

$$
\begin{aligned}
\Omega_{1} & =-\Omega_{\text {cor }}+\sqrt{\Omega_{\text {cor }}^{2}-\Omega_{n}^{2}}, \\
\Omega_{2} & =-\Omega_{\text {cor }}-\sqrt{\Omega_{\text {cor }}^{2}-\Omega_{n}^{2}}, \\
\Omega_{\text {cor }} & =i \frac{\bar{U} \zeta_{f} \lambda}{1+\zeta_{f}}, \\
\Omega_{n} & =\sqrt{\frac{\lambda^{4}-\lambda^{2} \bar{U}^{2} \zeta_{f}}{1+\zeta_{f}}}
\end{aligned}
$$

with the characteristic equation for the values of $\lambda$ at both ends as

$$
\tan \lambda-\frac{5}{3} \lambda=0
$$

and Appendix B gives the summary of the above results.

\section{Analysis of Results and Discussion}

Although the effect of Coriolis force on the dynamics of a fluid-conveying pipeline has been well known over the decades, early studies were shrouded in ad hoc heuristic arguments on the one hand and elegant interpretation of physical and numerical experiments on the other. The challenge has been to find a simple and straightforward way of demonstrating what has come to be accepted as the general pattern of behaviour of these systems.

In several investigations to date on the general behaviour and conditions of stability associated with unstable oscillation of pipe conveying fluid, researchers were preoccupied with finding answers to two basic questions, namely,

(a) constructing the general pattern of the dynamic response of the system,

(b) establishing the region and or regime where such a solution holds validity.

Within the context of linear theory, the latter question invariably involves determining the critical velocity of internal fluid flow for the system while the former exercise has unraveled curious and unexpected paradoxical patterns of flow.

Most of the difficulties encountered can be traced to the methodology used in tackling these problems wherein reliance was put on solving abridged equations whose solutions were fortified with powerful and masterly interpretation of physical and numerical experimental results.

In this short note we recovered some of the well-known results from a straightforward application of Fourier complex transform to the full linear equations. This affords us the opportunity to

(a) obtain closed form explicit expressions for classical flow variables such as the critical flow velocity and natural frequency of the system,

(b) avoid the tedium of laborious numerical work by being able to locate the eigenvalues of the critical flow velocity in a relatively simple fashion, 


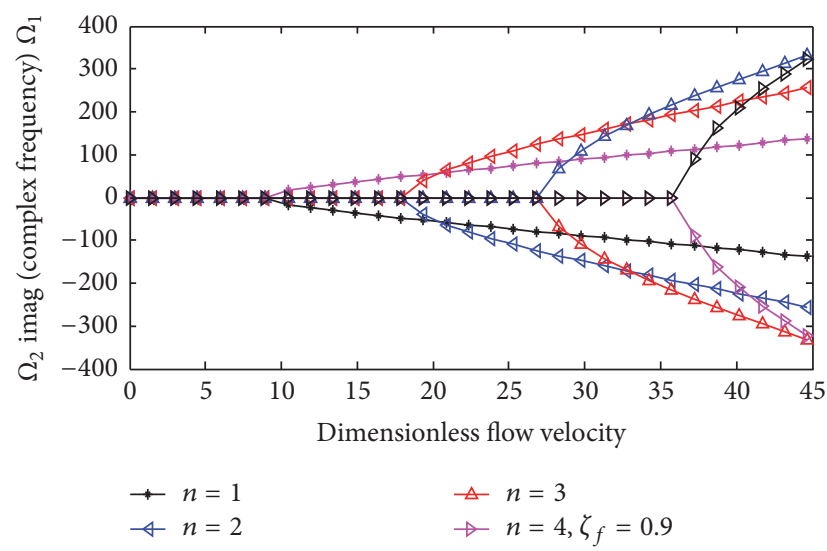

(a) Graph of $s=\operatorname{imag}\left(\Omega_{\mathrm{cor}} \pm \sqrt{\Omega_{\mathrm{cor}}^{2}-\Omega_{n}^{2}}\right)$

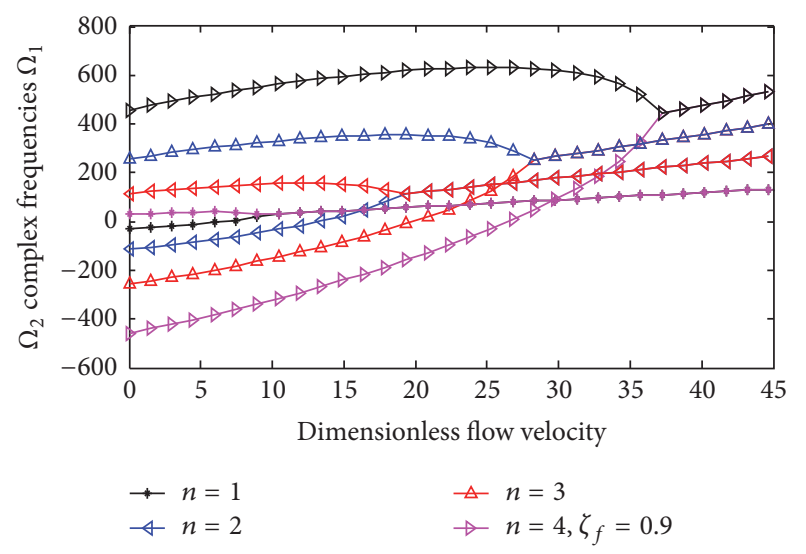

(b) Graph of $s=\operatorname{real}\left(\Omega_{\text {cor }} \pm \sqrt{\Omega_{\text {cor }}^{2}-\Omega_{n}^{2}}\right)$

FIGURE 4: Variation of complex frequency with internal axial flow velocity for different modes.

(c) undertake easy comparative parametric analysis of the variables contained in the solution without heavy reliance on numerical computation,

(d) guarantee, ab initio, that the derived solution complies with the exact or complete configuration and profile of the entire pipe length at onset so that the evolution of the final configuration as a direct result of the initial pipe profile does not constitute an issue,

(e) also provide confirmation of predictions of earlier workers and facilitates a better understanding, organization, and interpretation of some of the phenomena reported to date in the literature.

In respect of the case of a simply supported beam hanging above the ground, our investigation clearly shows from (26) that the frequency associated with the Coriolis force is partly driven by the mass ratio of the internal fluid and increases linearly with the flow velocity. The critical flow velocity is found to be dependent on the fluid-to-pipe mass ratio $\zeta_{f}$, thereby showing that it is a function of the mass of the fluid flowing in the pipe. Equations (47), (57), and (61) also demonstrated that the system's dynamic responses are dependent on the initial configurations of the pipeline system and the boundaries' forces and moments.

The profiles illustrated (Figures 4-6) confirmed the mirror imaging or characteristics for the real, imaginary, and absolute values of the complex frequencies. At the critical points, flow bifurcations are demonstrated. As for the absolute values of the complex frequency pairs, the critical values and the two regimes of the beam divergence are clearly represented in the figures. We have equally noted that, for a given mass ratio, critical flow velocities are ordered in consonant with the number of modes.

The family of curves for the natural frequency of the system are illustrated (Figures 7-9) as a function of the flow velocity and fluid-pipe mass ratio. With (34) and (52), the subcritical velocity points corresponding to the predivergence values of the system are shown by the curves representing the imaginary parts of $\Omega_{n}$ against the flow velocity $\bar{U}$ in Figures 7(a) and 8(a) and against mass fluid ratio $\zeta_{f}$ in Figure 9(a). Also, as described by (34) and (53) and Figures 7(b), 8(b), and 9(b), the postcritical velocity points corresponding to the postdivergence values are demonstrated for the real part of $\Omega_{n}$, while the curves for the absolute values of $\Omega_{n}$ as illustrated with (51) show clearly the critical points for both the flow velocity and the fluid mass ratio. The curves for both the imaginary and real parts of $\Omega_{n}$ appear to depict quasi-static configurations from zero flow velocity and mass fluid ratio to the neighbourhood of the critical points and from the same neighbourhood, such quasi-static behaviour is observable as the flow velocity and mass fluid ratio increases beyond the critical points, respectively.

From the foregoing, it does not sound reasonable and possible even from actual experiments for a buckled fluidconveying pipeline to exhibit a quasi-static geometrical configuration beyond the critical points ad infinitum as shown theoretically in literature. It therefore seems scientifically sensible to study the dynamic response and natural frequency characteristics of a pipeline from the subcritical through critical and postcritical points in order to clearly describe the behavioural divergences of the system.

As shown in Figures 1 and 10, buckling occurs even in the absence of flow for a pipeline segment simply supported at both ends. This condition arises from the deformation of the neutral axis of an originally straight beam, at equilibrium, but is subjected to the action of its weight and the two end reactions. Thus, for a pipeline segment closed at both ends that is carrying an entrained static fluid, the degree of buckling increases as the mass of internal flow increases. However, if dynamic buckling occurs when a fully developed fluid is flowing through the pipe under the same conditions at any flow velocity, the direction of buckling alternates different values of flow velocity as demonstrated in Dodds Jr. and Runyan (1965) [18] for the case of the principal buckling mode $n=1$ and the behavioural pattern of curves in Figure 10. As demonstrated in this figure, the curves describing the physics of dynamically excited simply supported pipeline systems are parabolas and then show that the downward sagging of the pipe for time $\bar{t}=0$ and as the pipe is dynamically moving due to the fluid 


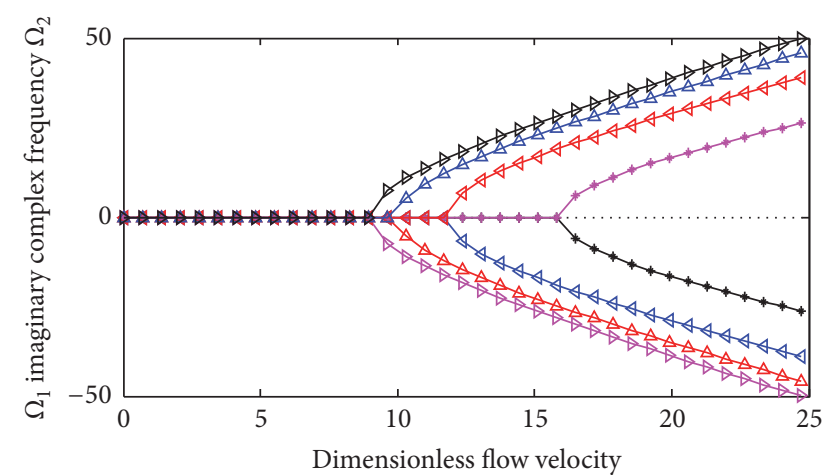

$$
\begin{array}{ll}
\rightarrow \zeta_{f}=0.1 & \triangle \zeta_{f}=0.3 \\
\triangleleft \zeta_{f}=0.2 & \rightarrow \zeta_{f}=0.4 ; n=1
\end{array}
$$

(a) Graph of $s=\operatorname{imag}\left(\Omega_{\text {cor }} \pm \sqrt{\Omega_{\text {cor }}^{2}-\Omega_{n}^{2}}\right)$

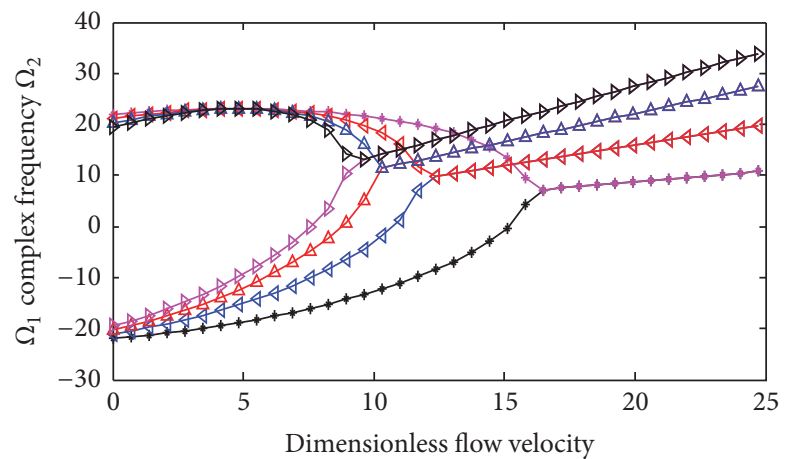

$$
\begin{array}{ll}
\rightarrow \zeta_{f}=0.1 & \rightarrow \zeta_{f}=0.3 \\
\rightarrow \zeta_{f}=0.2 & \rightarrow \zeta_{f}=0.4 ; n=1
\end{array}
$$

(b) Graph of $s=\operatorname{real}\left(\Omega_{\mathrm{cor}} \pm \sqrt{\Omega_{\mathrm{cor}}^{2}-\Omega_{n}^{2}}\right)$

FIGURE 5: Variation of complex frequency with axial flow velocity for different fluid mass ratios.

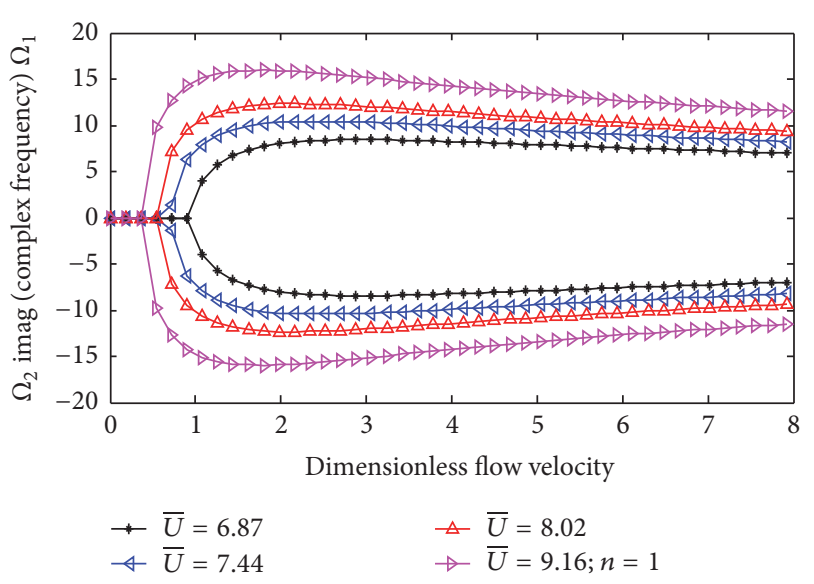

(a) Graph of $s_{1}=\operatorname{imag}\left(\Omega_{\text {cor }}+\sqrt{\Omega_{\text {cor }}^{2}-\Omega_{n}^{2}}\right)$

FIGURE 6: Movement of critical flow velocity as a function of fluid mass ratio for different axial flow velocities.

flow, depending on its magnitude, the pipe characteristics alternate to give convex and concave curvatures. More so, as interchange of energy develops between the generated Coriolis force and the system, symmetric and antisymmetric oscillations emerge. Also, the slow-moving frequency driven responses enveloping the fast-moving frequency driven ones are demonstrated in Figure 11, thus generating two distinct modes for the system.

As for Case 2 results, in Figures 12-17, the frequency curves for both the complex and natural frequencies are demonstrated to be similar to those for the simply supported pipe, except that their wave numbers are smaller $\lambda$ values and of complex forms as the transcendental characteristic equation is solved. The fluttering behaviours that are expected of cantilever systems are illustrated in Figures 15 and 16. This naturally explains the results that a pipe with a freeend gives. For these pipeline arrangements, the response-time characteristics shown in Figure 17 explain the envelopments of the fast-moving frequency responses by their slow-moving counterparts and that as time increases the amplitudes decay. These indeed show that the system performs what is known as beats phenomenon. These features are of paramount significance for design purposes.

Illustrated by Figures 18 and 19 are the results for Case 3, that is, the clamped-pinned pipeline segment. In the figures, the dynamic responses against the pipe lengths are clearly demonstrated as the clamped end reveals the zero gradients due to the moments and reactions at that end as well as zero moments and deflections at the simply supported end.

Case 1 Results. For responses of the pipe simply supported at both ends see Figures 4-11.

Case 2. For results of the cantilever pipe, see Figures 12-17.

Case 3 Results. For responses of the clamped-pinned ends pipe, see Figures 18 and 19.

\section{Conclusion}

The suitability of Fourier complex exponential transform method for the solution of the homogeneous Coriolis-term dependent mathematical physics equation governing the flow-induced vibration propelled by the conveyance of an incompressible fluid through a pipe segment is presented. The study assumed a linear theory for the fluid structure interaction mechanics where the relevant forces are properly accounted for.

Notably, this fluid structure interaction process as discussed in this paper has the tendency to induce the occurrences of conjugate modal complex and natural frequencies for all flow velocities and other associated parametric variables. This conjugateness of the complex frequencies is the prime sources for the initiation of bifurcation responses. Nevertheless, the modal natural frequencies as inferred in the investigation above can be functionally related to the Coriolis frequency. It is instructive to state that their lines 


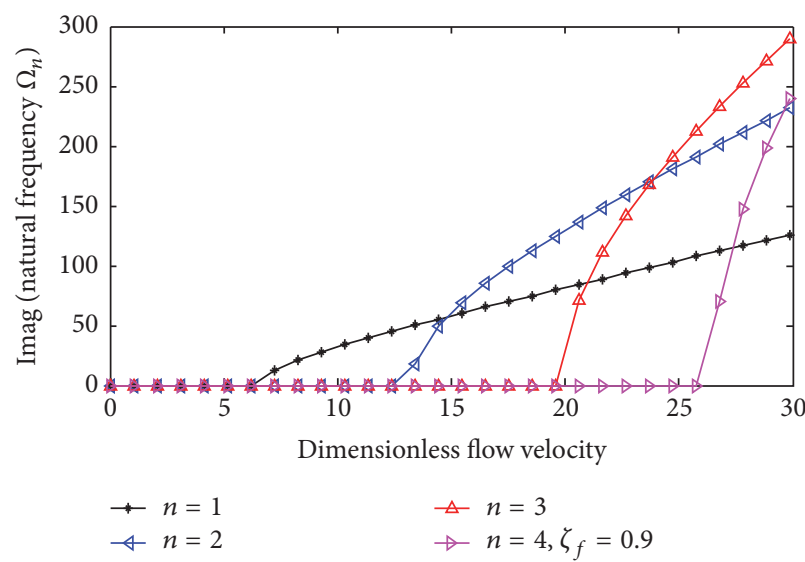

(a) Graph of imag $\left(\Omega_{n}\right)$

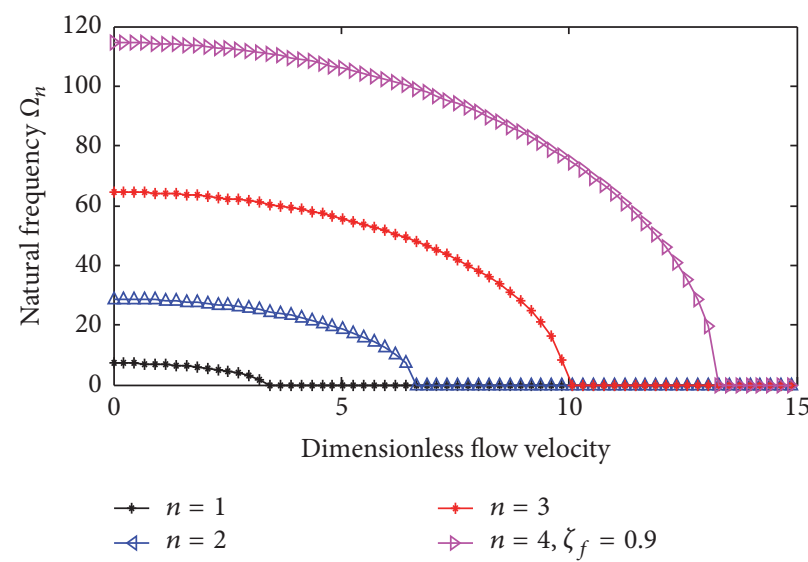

(b) Graph of real $\left(\Omega_{n}\right)$

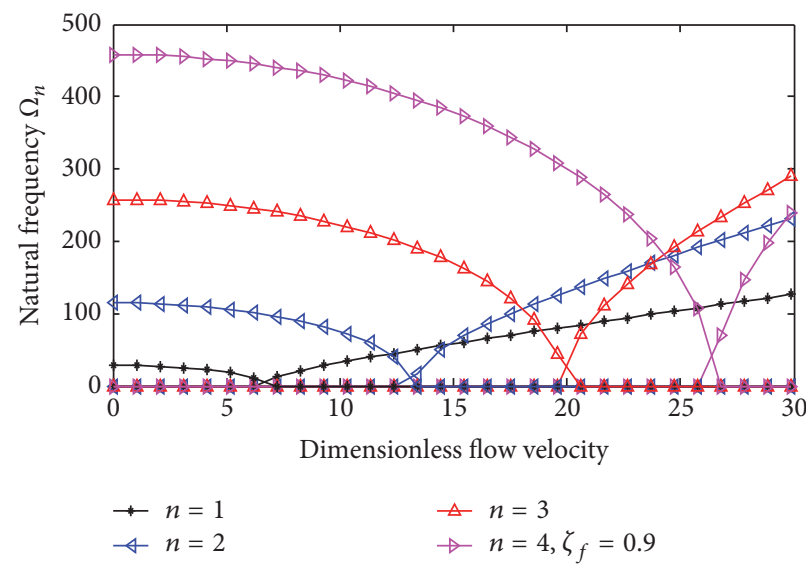

(c) Graph of absolute $\left(\Omega_{n}\right)$

Figure 7: Variation of natural frequency with internal axial flow velocity for different modes.

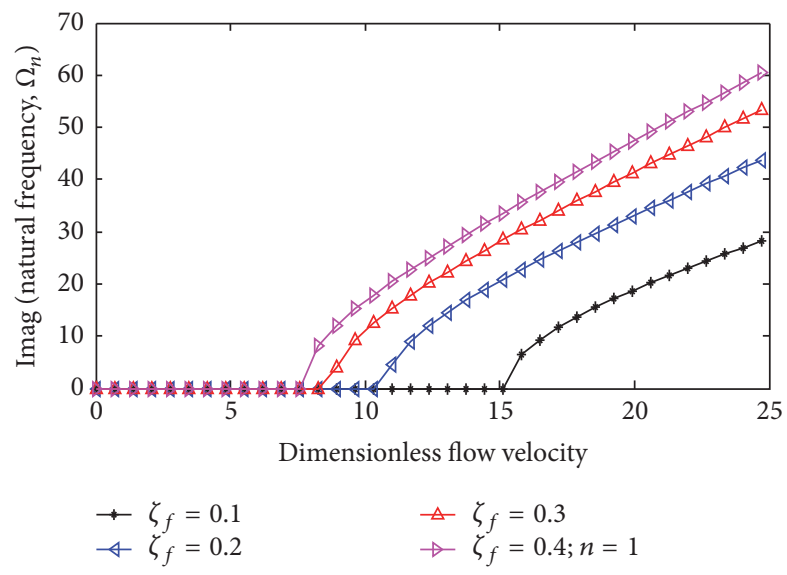

(a) Graph of imag $\left(\Omega_{n}\right)$

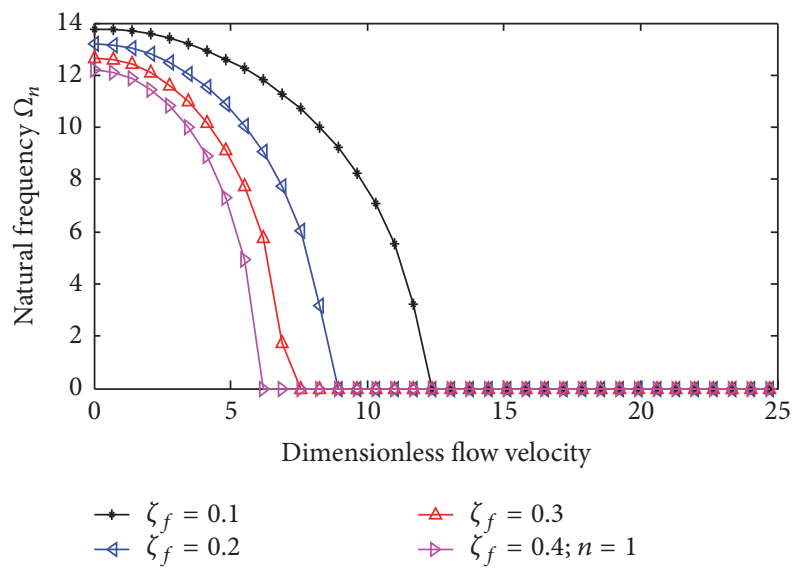

(b) Graph of real $\left(\Omega_{n}\right)$

FIGURE 8: Variation of natural frequency with axial flow velocity for different fluid mass ratios.

of action are directed orthogonally to the directrices of the complex frequencies where the Coriolis force is playing a central role. It is also noteworthy to state that whilst the modal natural frequency equation can be alternatively conjured to be independent of Coriolis force as an option, the dynamic responses and complex frequencies are seen to be explicitly tied to the modulating roles of the Coriolis acceleration. Consequently, the use of this transform method can be very apt to conjure approximate closed form solutions for nonlinear problems in conjunction with homotopy perturbation 


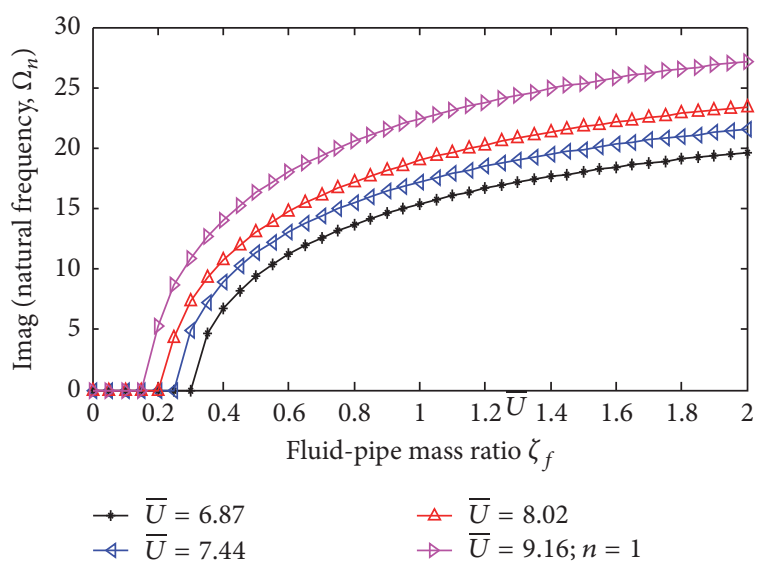

(a) Graph of $\operatorname{imag}\left(\Omega_{n}\right)$

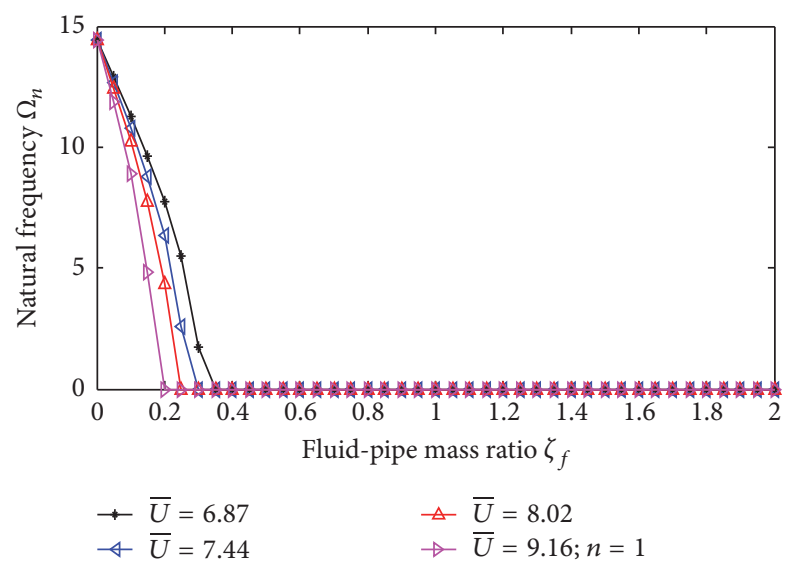

(b) Graph of $\operatorname{real}\left(\Omega_{n}\right)$

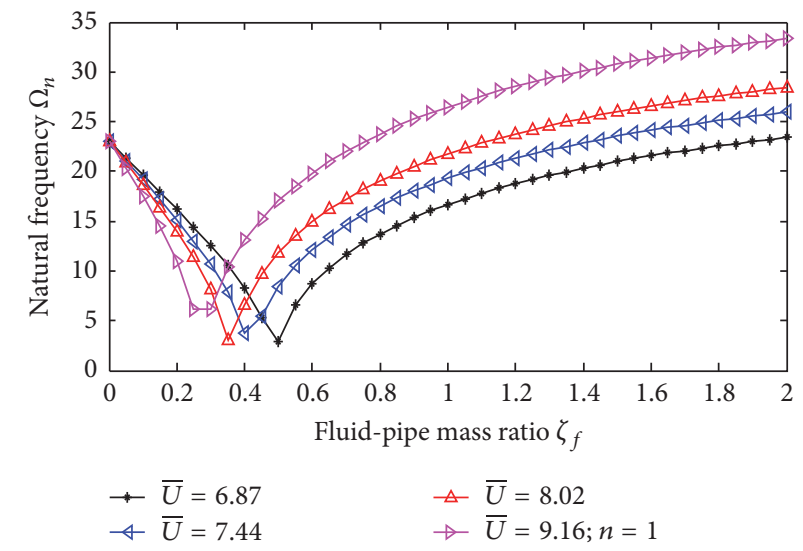

(c) Graph of $\left\|\Omega_{n}\right\|$

FIgURE 9: Movement of critical flow velocity as a function of fluid mass ratio for different axial flow velocities.

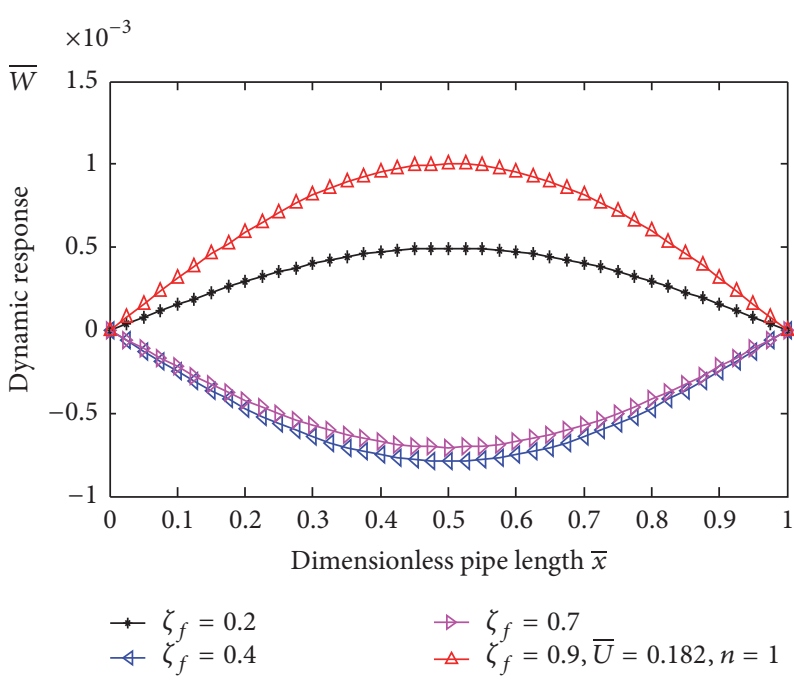

Figure 10: Dynamic response of pipe as a function of mass ratio.

method (HPM), homotopy analysis method, and differential transformation method (DTM), without having recourse to the uses of singular or parameter perturbation method where

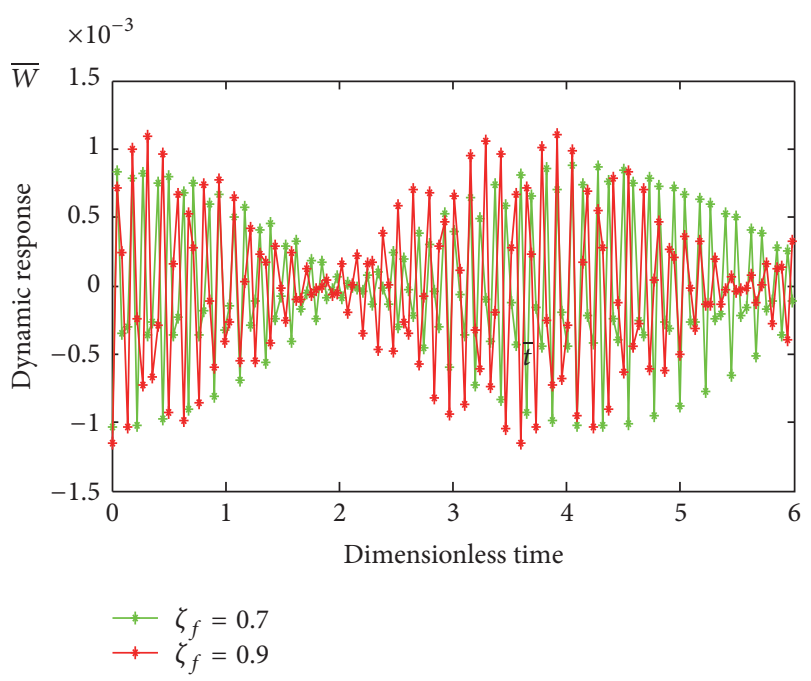

FIGURE 11: Graph of response against dimensionless time for given fluid-pipe mass ratios.

the issue of smaller parameters for the perturbation series expansions is an issue. 

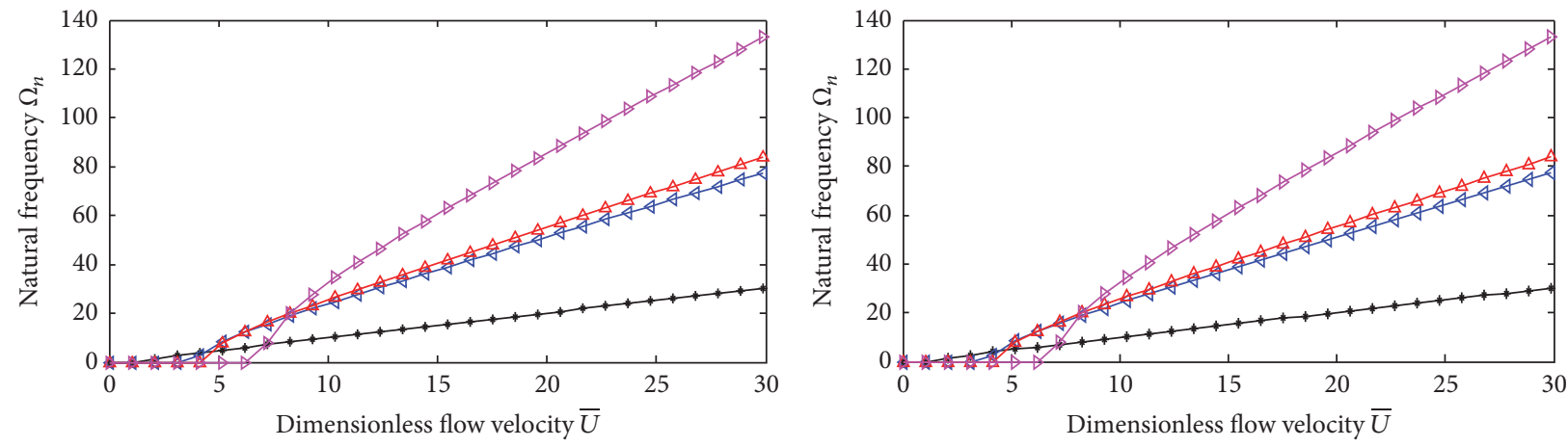

$\begin{array}{ll}\rightarrow \lambda=1.47 & \triangle \lambda=4.12 \\ \leftarrow \lambda=3.80 & \rightarrow \lambda=6.65^{\prime} \zeta_{f}=0.9\end{array}$

(a)

$$
\begin{aligned}
& \rightarrow \lambda=1.47 \\
& \leftarrow \lambda=3.80
\end{aligned}
$$

(b)

FIGURE 12: (a) Graphs of natural frequency versus flow velocity. (b) Graphs of natural frequency versus flow velocity.

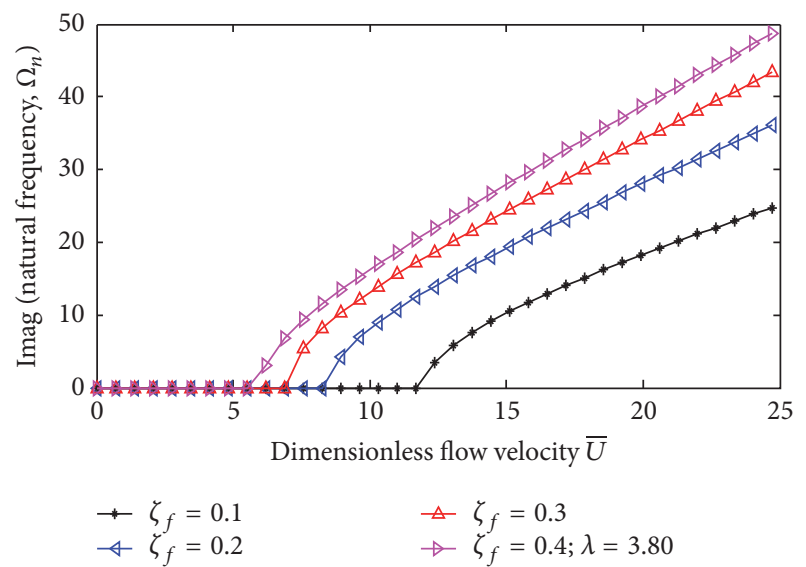

(a)

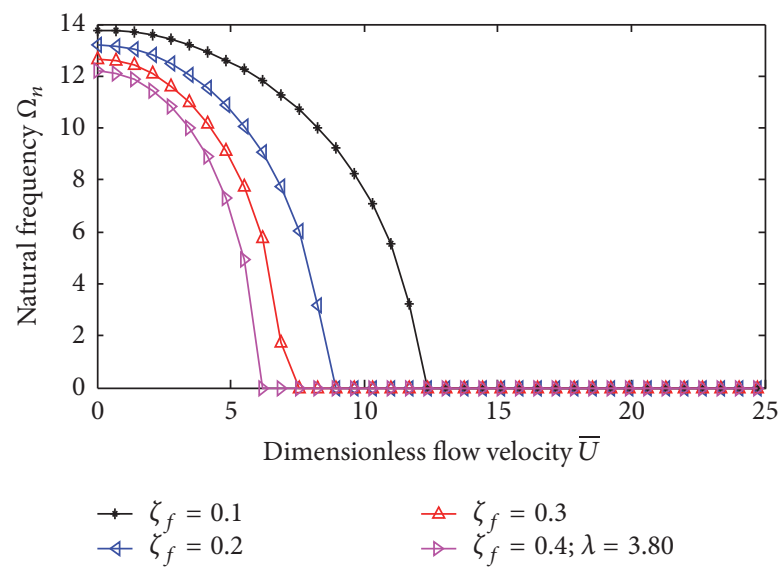

(b)

FIGURE 13: (a) Graphs of natural frequency versus flow velocity. (b) Graphs of natural frequency versus flow velocity.

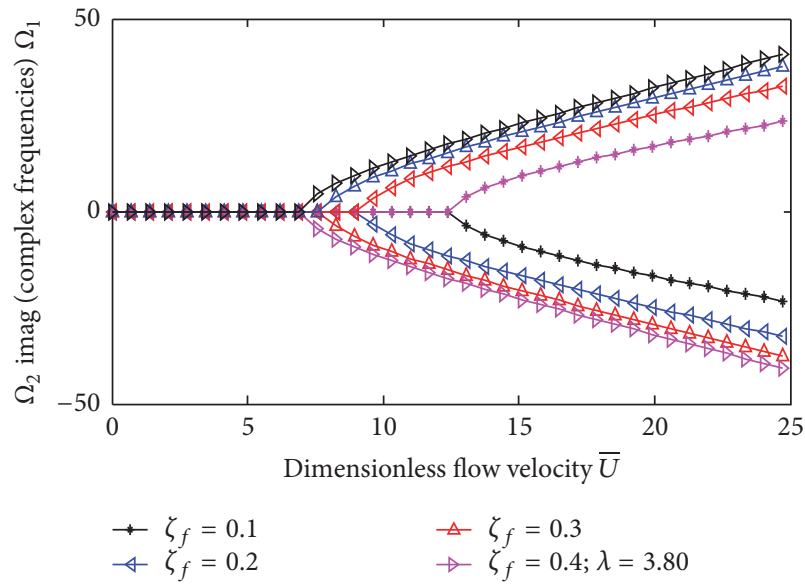

(a) Graph of imaginary $\Omega_{1}$ and $\Omega_{2}$

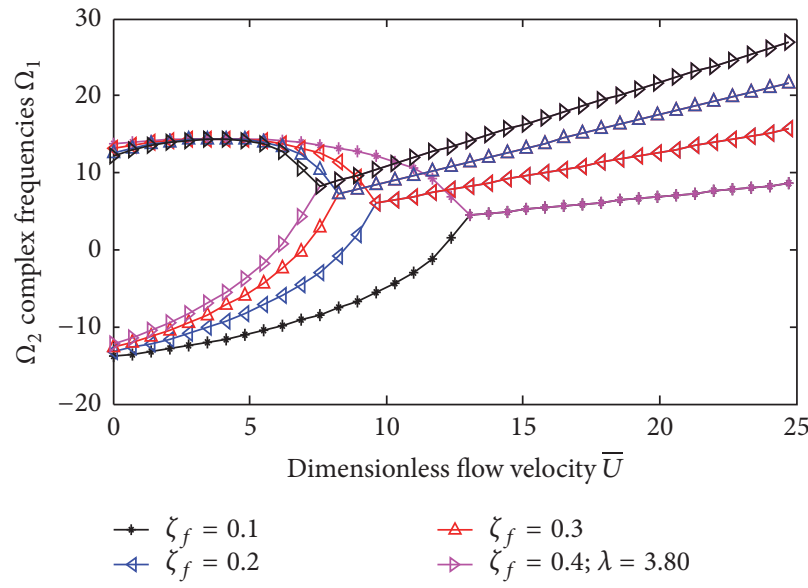

(b) Graph of $\Omega_{1}$ and $\Omega_{2}$

FIGURE 14: Graphs of complex frequency versus flow velocity. 


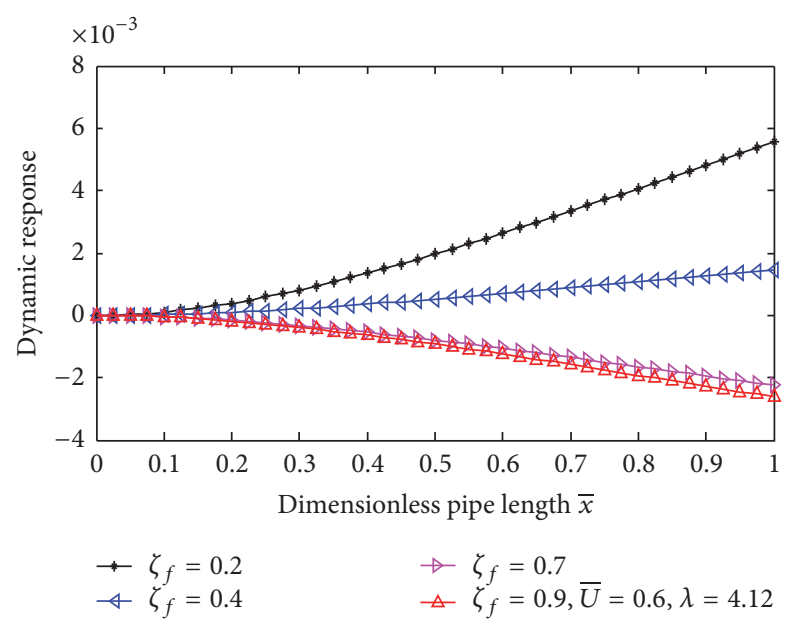

FIGURE 15: Dynamic response of pipe of a given internal flow velocity and different mass ratio.

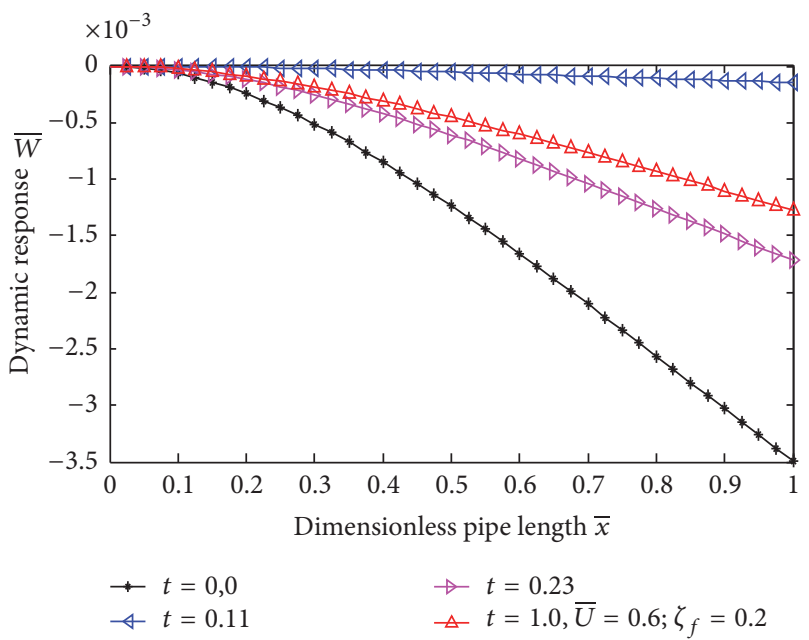

FIGURE 16: Dynamic response of pipe of a given mass ratio for different time.

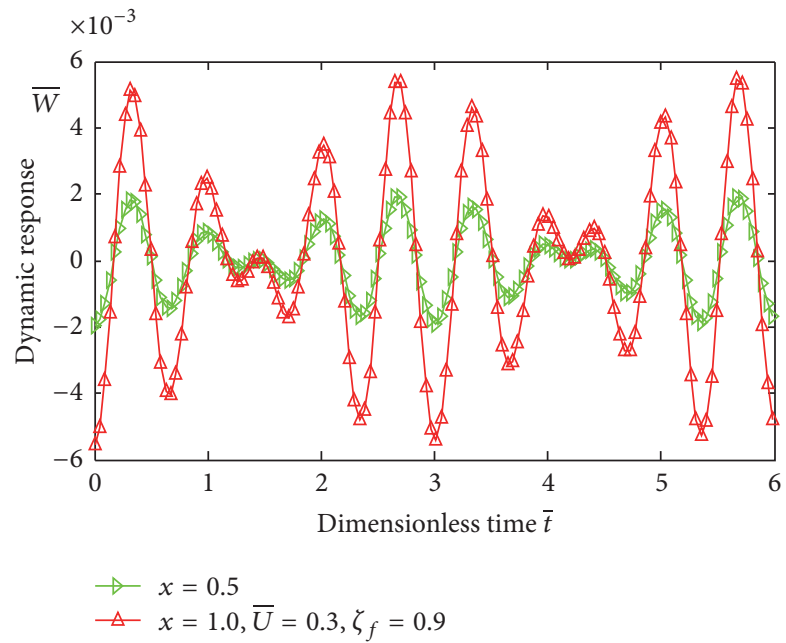

FIgURE 17: Dynamic response of pipe at $x=0.5$ and 1.0 for a given axial flow velocity and fluid-pipe mass ratio of 0.9 .

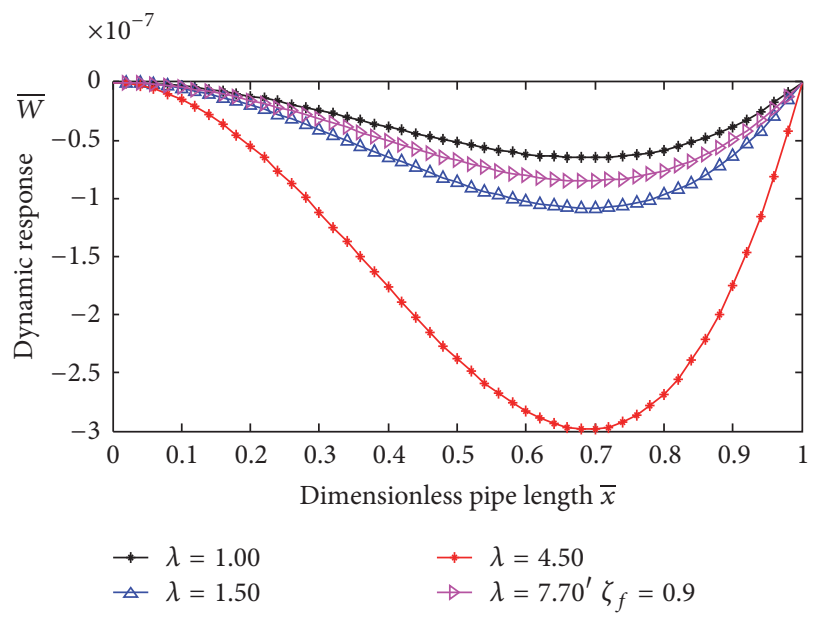

FIGURE 18: Dynamic response of pipe of a given mass ratio for different wave numbers.

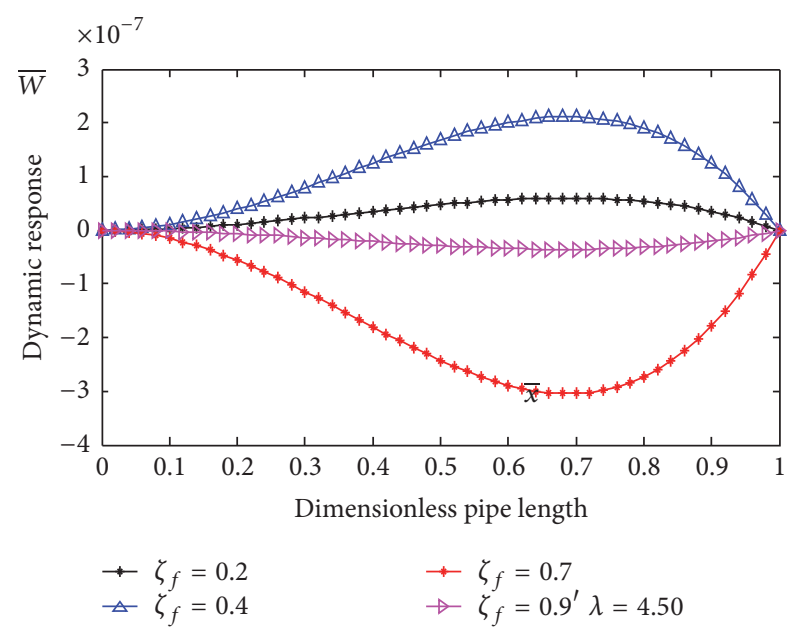

FIgURE 19: Dynamic response of pipe of a given value of $\lambda$ for different mass ratios.

\section{Appendix}

\section{A. Critical Flow Velocity}

For Case 1, a pipe simply supported at both ends has physical configuration as shown schematically in Figure 20. Since the pipe and the fluid it is carrying have uniformly distributed downward vertical loads of intensity $f$ on the system, then we consider the shearing force $F$ at a distance $\bar{x}$ from the left end; it is given as shown in Figure 20.

The deflection function is given as

$$
\bar{W}=\bar{w}\left(\frac{\bar{x}^{3}}{12}-\frac{\bar{x}^{4}}{24}-\frac{\bar{x}}{24}\right),
$$

where $\bar{w}=\beta_{f}\left(1+\zeta_{f}\right)$ and $\beta_{f}=m_{p} L / 2 E \widehat{I}$. 


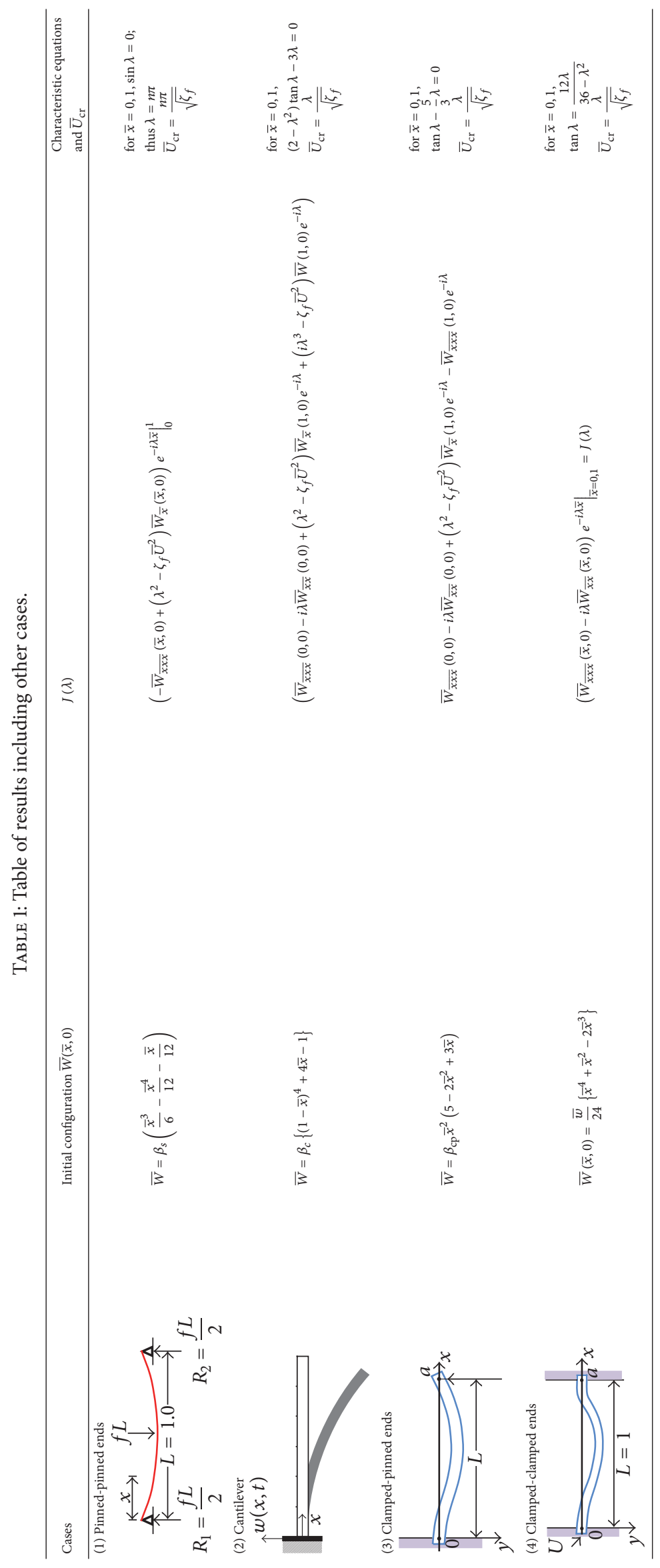




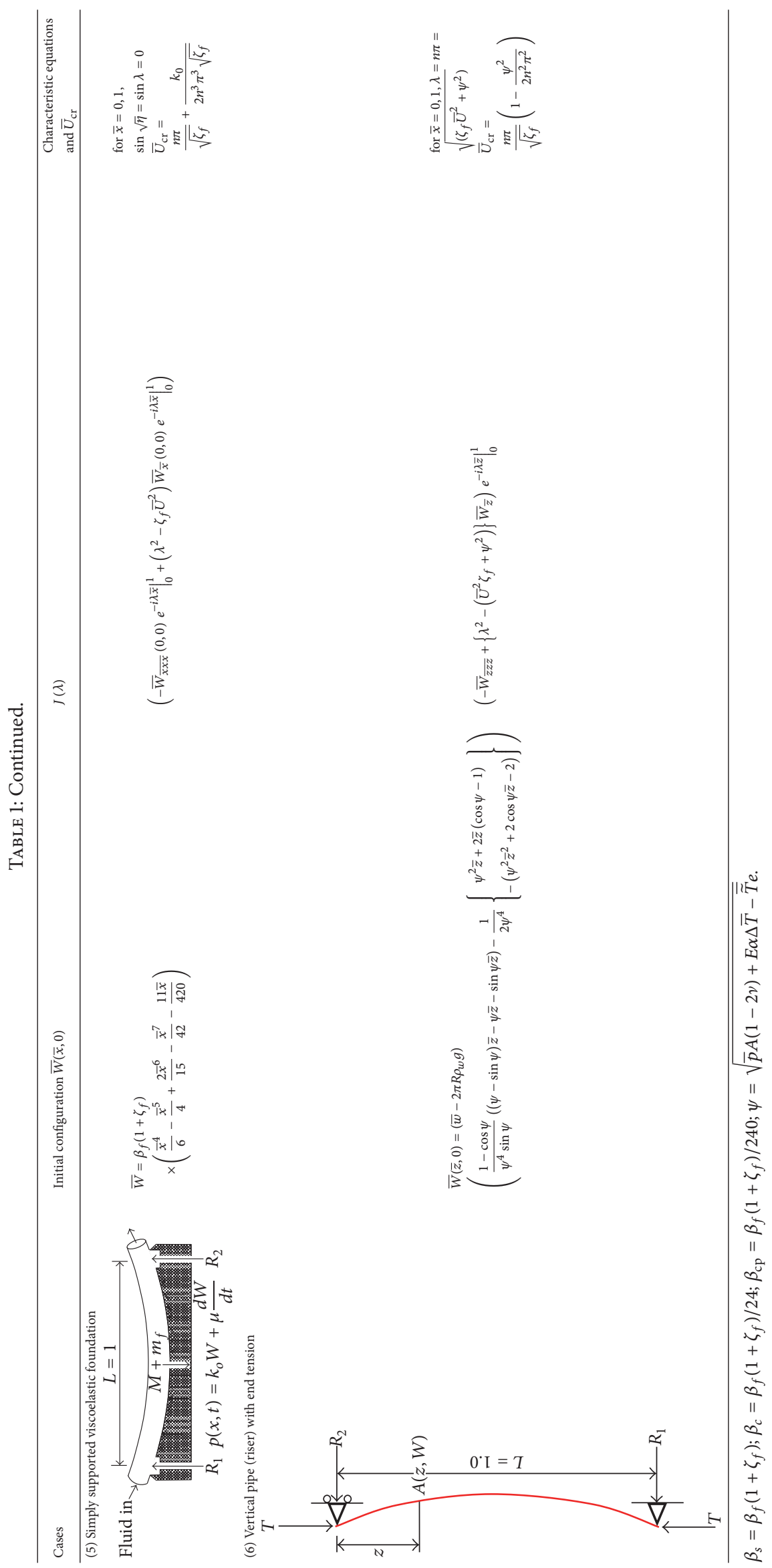




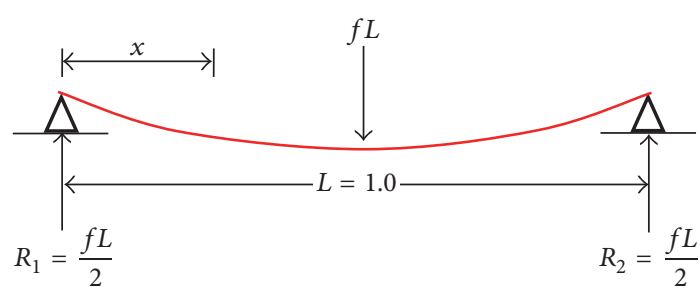

FIGURE 20: Simply supported beam.

In this case, while the shearing force varies linearly, the bending moment varies parabolically along the length of the beam.

$$
\begin{aligned}
\bar{W}(\bar{x}, \bar{t}) & \left\{\bar{w}\left(\frac{\left(\Omega_{2} e^{-i \Omega_{1} \bar{t}}-\Omega_{1} e^{-i \Omega_{2} \bar{t}}\right)-i 2 \Omega_{\text {cor }}\left(e^{-i \Omega_{1} \bar{t}}-e^{-i \Omega_{2} \bar{t}}\right)}{\left(\Omega_{2}-\Omega_{1}\right)}\right)\right. \\
& \cdot\left(\frac{\bar{x}^{4}}{24}-\frac{\bar{x}^{3}}{12}+\frac{\bar{x}}{24}\right)-\frac{\left(\Omega_{1}\left(e^{-i \Omega_{2} \bar{t}}-1\right)-\Omega_{2}\left(e^{-i \Omega_{1} \bar{t}}-1\right)\right)}{\left(\Omega_{1}-\Omega_{2}\right)} \\
& \left.\cdot \int_{0}^{\infty} \frac{J_{s s}(\lambda)}{\Omega_{n}^{2}} d \lambda\right\} ;
\end{aligned}
$$

that is,

$\bar{W}(\bar{x}, \bar{t})$

$$
\begin{aligned}
& =\bar{w}\left\{\left(\frac{\left(\Omega_{2} e^{-i \Omega_{1} \bar{t}}-\Omega_{1} e^{-i \Omega_{2} \bar{t}}\right)-i 2 \Omega_{\mathrm{cor}}\left(e^{-i \Omega_{1} \bar{t}}-e^{-i \Omega_{2} \bar{t}}\right)}{\left(\Omega_{2}-\Omega_{1}\right)}\right)\right. \\
& \cdot\left(\frac{\bar{x}^{4}}{24}-\frac{\bar{x}^{3}}{12}+\frac{\bar{x}}{24}\right)-\frac{\left(\Omega_{1}\left(e^{-i \Omega_{2} \bar{t}}-1\right)-\Omega_{2}\left(e^{-i \Omega_{1} \bar{t}}-1\right)\right)}{12\left(\Omega_{1}-\Omega_{2}\right)} \\
& \left.\cdot \int_{0}^{\infty} \frac{\left\{\left(\lambda^{2}-\zeta_{f} \bar{U}^{2}-12\right)\left(e^{-i \lambda(1-\bar{x})}+e^{i \lambda \bar{x}}\right)\right\}}{\Omega_{n}^{2}} d \lambda\right\} .
\end{aligned}
$$

But with the boundary conditions, that is, $\bar{W}(0, \bar{t})=\bar{W}(1, \bar{t})=$ 0 , the above equation shows that

$$
\begin{aligned}
& \bar{w}\left(\frac{\left(\Omega_{2} e^{-i \Omega_{1} \bar{t}}-\Omega_{1} e^{-i \Omega_{2} \bar{t}}\right)-i 2 \Omega_{\mathrm{cor}}\left(e^{-i \Omega_{1} \bar{t}}-e^{-i \Omega_{2} \bar{t}}\right)}{\left(\Omega_{2}-\Omega_{1}\right)}\right) \\
& \cdot\left(\frac{\bar{x}^{4}}{24}-\frac{\bar{x}^{3}}{12}+\frac{\bar{x}}{24}\right)=0
\end{aligned}
$$

and thus the second term must be zero also; that is,

$$
-\int_{0}^{\infty} \frac{\left(\Omega_{1}\left(e^{-i \Omega_{2} \bar{t}}-1\right)-\Omega_{2}\left(e^{-i \Omega_{1} \bar{t}}-1\right)\right)}{12\left(\Omega_{1}-\Omega_{2}\right)} \frac{\left\{12\left(2 e^{-i \lambda(1-\bar{x})}+e^{i \lambda \bar{x}}\right)+\left(\lambda^{2}-\zeta_{f} \bar{U}^{2}\right)\left(e^{-i \lambda(1-\bar{x})}+e^{i \lambda \bar{x}}\right)\right\}}{\Omega_{n}^{2}} d \lambda=0
$$

That is, for all $t$,

$$
\int_{0}^{\infty} \frac{\left(\Omega_{1}\left(e^{-i \Omega_{2} \bar{t}}-1\right)-\Omega_{2}\left(e^{-i \Omega_{1} \bar{t}}-1\right)\right)}{12\left(\Omega_{1}-\Omega_{2}\right)} \frac{\left\{12\left(2 e^{-i \lambda(1-\bar{x})}+e^{i \lambda \bar{x}}\right)+\left(\lambda^{2}-\zeta_{f} \bar{U}^{2}\right)\left(e^{-i \lambda(1-\bar{x})}+e^{i \lambda \bar{x}}\right)\right\}}{\left(\lambda^{4}-\lambda^{2} \bar{U}^{2} \zeta_{f}\right)} d \lambda=0
$$

becoming

$$
\int_{0}^{\infty} \frac{\left(\Omega_{1}\left(e^{-i \Omega_{2} \bar{t}}-1\right)-\Omega_{2}\left(e^{-i \Omega_{1} \bar{t}}-1\right)\right)}{12\left(\Omega_{1}-\Omega_{2}\right)} \frac{\left\{12\left(2 e^{-i \lambda(1-\bar{x})}+e^{i \lambda \bar{x}}\right)+\left(\lambda^{2}-\zeta_{f} \bar{U}^{2}\right)\left(e^{-i \lambda(1-\bar{x})}+e^{i \lambda \bar{x}}\right)\right\}}{\left(\lambda-\sqrt{\eta_{1}}\right)\left(\lambda+\sqrt{\eta_{1}}\right)} d \lambda=0
$$

where

Using residue theory and contour integration, we deduce that

$$
\eta_{1}=\frac{\bar{U}^{2} \zeta_{f}}{2} \pm \sqrt{\frac{\bar{U}^{4} \zeta_{f}^{2}}{2}}
$$

$$
\begin{gathered}
\lambda=\sqrt{\eta_{1}} \\
\text { or } \lambda= \pm \bar{U} \sqrt{\zeta_{f}} .
\end{gathered}
$$


Adding the residues of (A.7) gives

$$
\begin{array}{r}
\frac{12}{2 \sqrt{\eta_{1}}}\left\{2\left(e^{-i \sqrt{\eta_{1}}(1-\bar{x})}-e^{i \sqrt{\eta_{1}}(1-\bar{x})}\right)\right. \\
\left.+\left(e^{i \sqrt{\eta_{1} x}}-e^{-i \sqrt{\eta_{1} x}}\right)\right\}\left.\right|_{\bar{x}=0,1}=0,
\end{array}
$$

that is,

$$
\left.i \frac{12}{\sqrt{\eta_{1}}}\left\{-2 \sin \sqrt{\eta_{1}}(1-\bar{x})+\sin \sqrt{\eta_{1} x}\right\}\right|_{\bar{x}=0,1}=0
$$

so that when $\bar{x}=0$, at one end, then,

$$
\sin \sqrt{\eta_{1}}=0, \quad \forall \sqrt{\eta_{1}}=\lambda=n \pi
$$

Similarly with $\bar{x}=1$, at the other end, therefore the critical velocity is given as

$$
\bar{U}_{\text {critical }}=\frac{n \pi}{\sqrt{\zeta_{f}}} \quad \forall n=1,2, \ldots
$$

and the dynamic response becomes

$$
\begin{aligned}
& \bar{W}(\bar{x}, \bar{t}) \\
& =\bar{w}\left\{\left(\frac{\left(\Omega_{2} e^{-i \Omega_{1} \bar{t}}-\Omega_{1} e^{-i \Omega_{2} \bar{t}}\right)-i 2 \Omega_{\mathrm{cor}}\left(e^{-i \Omega_{1} \bar{t}}-e^{-i \Omega_{2} \bar{t}}\right)}{\left(\Omega_{2}-\Omega_{1}\right)}\right)\right. \\
& \cdot\left(\frac{\bar{x}^{4}}{24}-\frac{\bar{x}^{3}}{12}+\frac{\bar{x}}{24}\right)+i \\
& \quad \frac{\left(\Omega_{1}\left(e^{-i \Omega_{2} \bar{t}}-1\right)-\Omega_{2}\left(e^{-i \Omega_{1} \bar{t}}-1\right)\right)}{n \pi\left(\Omega_{1}-\Omega_{2}\right)}\{2 \sin n \pi(1-\bar{x}) \\
& -\sin n \pi \bar{x}\}
\end{aligned}
$$

\section{B. Summary of Derived Results}

See Table 1.

\section{Nomenclature}

$$
\begin{array}{ll}
E: & \text { Young's modulus } \\
I: & \text { Moment of inertia } \\
\rho_{s}: & \text { Mass density of pipe per unit length } \\
\rho_{f}: & \text { Mass density of fluid per unit length } \\
\bar{U}: & \text { Internal flow velocity } \\
w: & \text { Transverse deflection } \\
\bar{W}: & \text { Dimensionless dynamic response } \\
\bar{W}_{\bar{x}}: & \text { Pipe's end slope } \\
\bar{W}_{\overline{x x}}: & \text { Pipe's end moment } \\
\bar{W}_{\overline{x x x}}: & \text { Pipe's end shear force } \\
\bar{t}: & \text { Dimensionless time } \\
\bar{x}: & \text { Coordinate of pipe's neutral axis } \\
g: & \text { Gravitational acceleration } \\
L: & \text { Pipe length }
\end{array}
$$

$\lambda$ : Wave number

$\zeta_{f}: \quad$ Fluid-pipe mass ratio

$\Omega_{0}$ : System frequency at zero flow velocity

$\Omega_{n}$ : Natural frequency

$\Omega_{1}$ : Complex frequency

$\Omega_{2}$ : Conjugate complex frequency

$\bar{p}: \quad$ Dimensionless pressure

$\bar{T}: \quad$ Dimensionless temperature

$v$ : Poison ratio

$\alpha$ : Linear expansivity

$\bar{p}: \quad$ Dimensionless pressure

$\Omega_{\text {cor }}$ : Frequency due to Coriolis force

$\bar{U}_{\mathrm{cr}}$ : Critical flow velocity.

\section{Conflicts of Interest}

The authors declare that they have no conflicts of interest.

\section{References}

[1] M. P. Paidoussis, Fluid-Structure Interactions: Slender Structures and Axial Flows, vol. 1, Academic Press, 2014.

[2] I. Elishakoff, "Controversy associated with the so-called 'follower forces': critical overview," Applied Mechanics Reviews, vol. 58, no. 1-6, pp. 117-142, 2005.

[3] H. R. Öz and H. Boyaci, "Transverse vibrations of tensioned pipes conveying fluid with time-dependent velocity," Journal of Sound and Vibration, vol. 236, no. 2, pp. 259-276, 2000.

[4] T. Szmidt and P. Przybyłowicz, "Critical flow velocity in a pipe with electromagnetic actuators," Journal of Theoretical and Applied Mechanics, vol. 51, no. 2, pp. 487-496, 2013.

[5] A. Askarian, H. Abtahi, and H. Haddaapour, "Dynamic instability of cantilevered composite pipe conveying flow with an end nozzle," in Proceedings of the International Congress on Sound and Vibration (ICSV'14), Beijing, China, 2014.

[6] G. L. Kuiper and A. V. Metrikine, "On stability of a clampedpinned pipe conveying fluid," HEBRON, vol. 49, no. 3, 2004.

[7] K. R. Chellapilla and H. S. Simha, "Vibrations of fluidconveying pipes resting on two-parameter foundation," The Open Acoustics Journal, vol. 1, pp. 24-33, 2008.

[8] J. Leklong, S. Chucheepsakul, and S. Kaewunruen, "Dynamic responses of marine risers/pipes transporting fluid subject to top end excitations," in Proceedings of the 8th ISOPE Pacific/Asia Offshore Mechanics Symposium, Bangkok, Thailand, 2008.

[9] A. H. Al-Hilli and T. J. Ntayeesh, "Free vibration characteristics of elastically supported pipe conveying fluid," Nahrain University College of Engineering Journal, vol. 16, no. 1, pp. 9-19, 2013.

[10] Q. Guo, L. Zhang, and L. Xiao, "Damage analysis of the vehicle's pipe conveying fluid induced by complex random-shock loads," Journal of Pressure Equipment and Systems, vol. 4, pp. 100-103, 2006.

[11] Y. L. Zhang, D. G. Gorman, J. M. Reese, and J. Horacek, "Observations on the vibration of axially tensioned elastomeric pipes conveying fluid," Proceedings of the Institution of Mechanical Engineers, Part C: Journal of Mechanical Engineering Science, vol. 214, no. 3, pp. 423-433, 2000.

[12] Y. Modarres-Sadeghi and M. P. Païdoussis, "Nonlinear dynamics of extensible fluid-conveying pipes, supported at both ends," Journal of Fluids and Structures, vol. 25, no. 3, pp. 535-543, 2009. 
[13] R. A. Ibrahim, "Overview of mechanics of pipes conveying fluids-part I: fundamental studies," Journal of Pressure Vessel Technology, vol. 132, no. 3, Article ID 034001, 32 pages, 2010.

[14] M. Murai and M. Yamamoto, "An experimental analysis of the internal flow effects on marine risers," in Proceedings of the MARTEC, BUET, pp. 159-165, Dhaka, Bangladesh, 2010.

[15] N. Marakala, K. Appukutttan, and R. Kadoli, "Experimental and theoretical investigation of combined effects of fluid and thermal induced vibration on vertical thin slender tube," IOSR Journal of Mechanical and Civil Engineering (IOSR-JMCE), pp. 63-68, 2014.

[16] U. Lee and J. Park, "Spectral element modelling and analysis of a pipeline conveying internal unsteady fluid," Journal of Fluids and Structures, vol. 22, no. 2, pp. 273-292, 2006.

[17] N. Qiao, W. Lin, and Q. Qin, "Bifurcations and chaotic motions of a curved pipe conveying fluid with nonlinear constraints," Computers and Structures, vol. 84, no. 10-11, pp. 708-717, 2006.

[18] H. L. Dodds Jr. and H. L. Runyan, "Effect of high velocity fluid flow on the bending vibrations and static divergence of a simplysupported pipe," NASA Technical Note D-2870, 1965.

[19] R. Yamaguchi, G. Tanaka, H. Liu, and T. Hayase, "Fluid vibration induced in T-junction with double side branches," World Journal of Mechanics, vol. 6, no. 4, pp. 169-179, 2016.

[20] J. Kutin and I. Bajsić, "Fluid-dynamic loading of pipes conveying fluid with a laminar mean-flow velocity profile," Journal of Fluids and Structures, vol. 50, pp. 171-183, 2014.

[21] M. J. Jweeg and T. J. Ntayeesh, "Dynamic analysis of pipes conveying fluid using analytical, numerical and experimental verification with the aid of smart materials," International Journal of Science and Research, vol. 4, no. 12, 2015.

[22] R. C. Wrede and M. Spiegel, Thoery and Problems of Advanced Calculus, Schaum's Outline Series, McGraw-Hill, New York, NY, USA, 2nd edition, 2002.

[23] P. S. Olayiwola, "Mechanics of a fluid-conveying pipeline system resting on a viscoelastic foundation," Journal of Multidisciplinary Engineering Science Studies (JMESS), vol. 2, no. 3, 2016.

[24] A. Jeffrey, Advanced Engineering Mathematics, Harcourt Academic Press, Burlington, Mass, USA, 2002.

[25] W. A. Nash, Theory and Problems of Strength of Materials, vol. 83 of Schaum's Outline Series, McGraw-Hill, 2nd edition, 1977. 


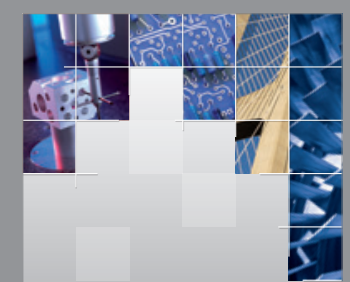

\section{Enfincering}
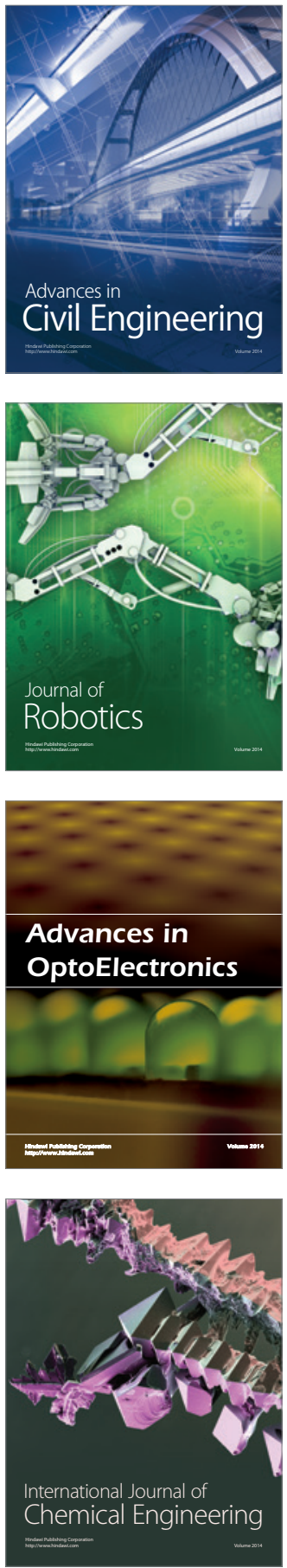

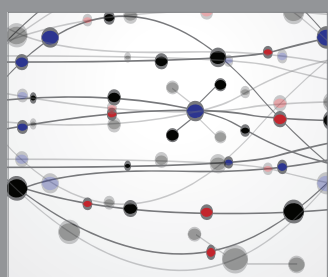

The Scientific World Journal

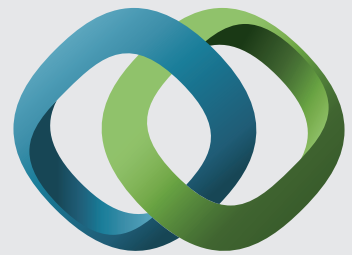

\section{Hindawi}

Submit your manuscripts at

https://www.hindawi.com
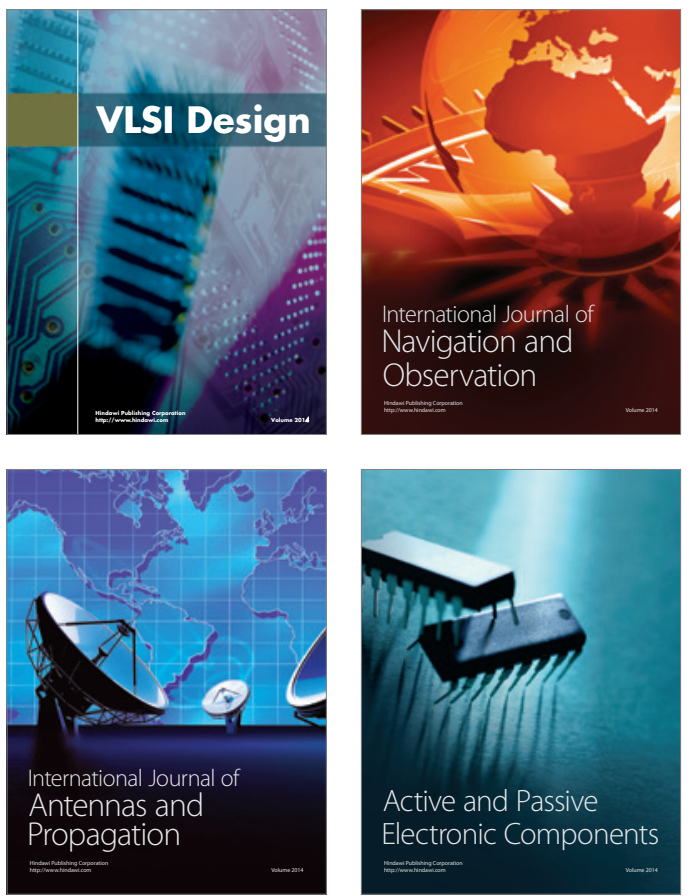
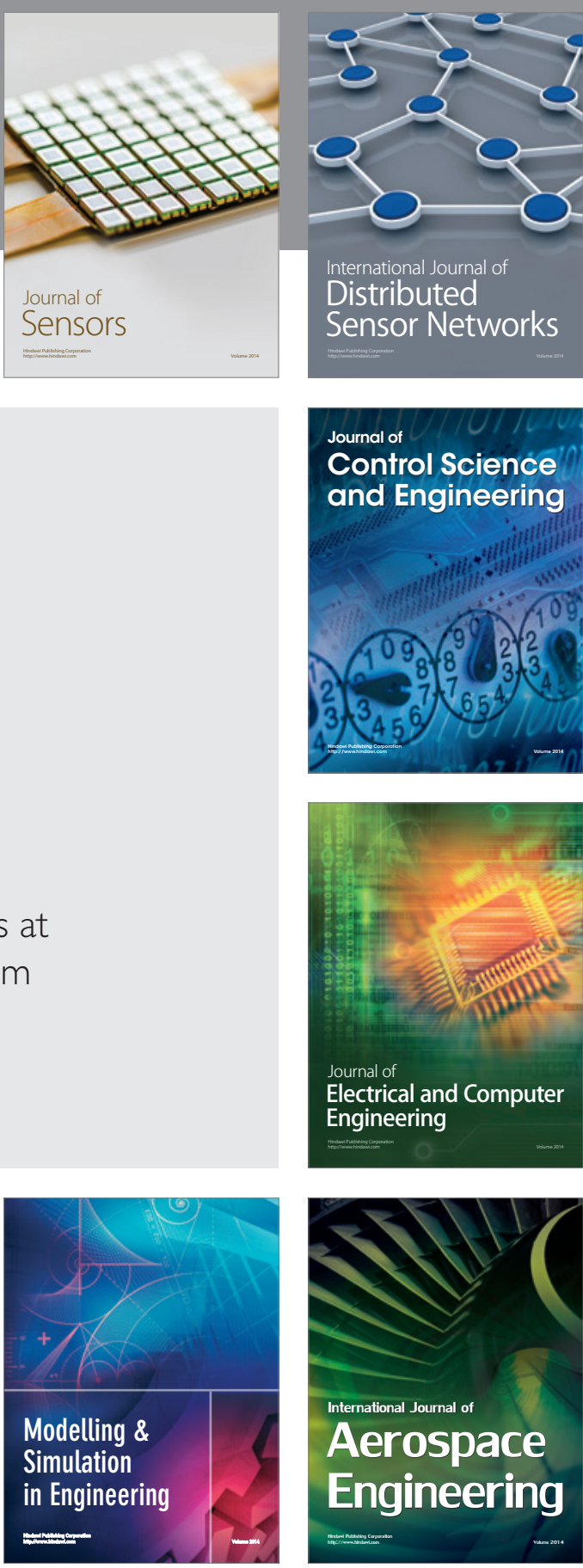

International Journal of

Distributed

Sensor Networks

$-$

Joumal of

Control Science

and Engineering
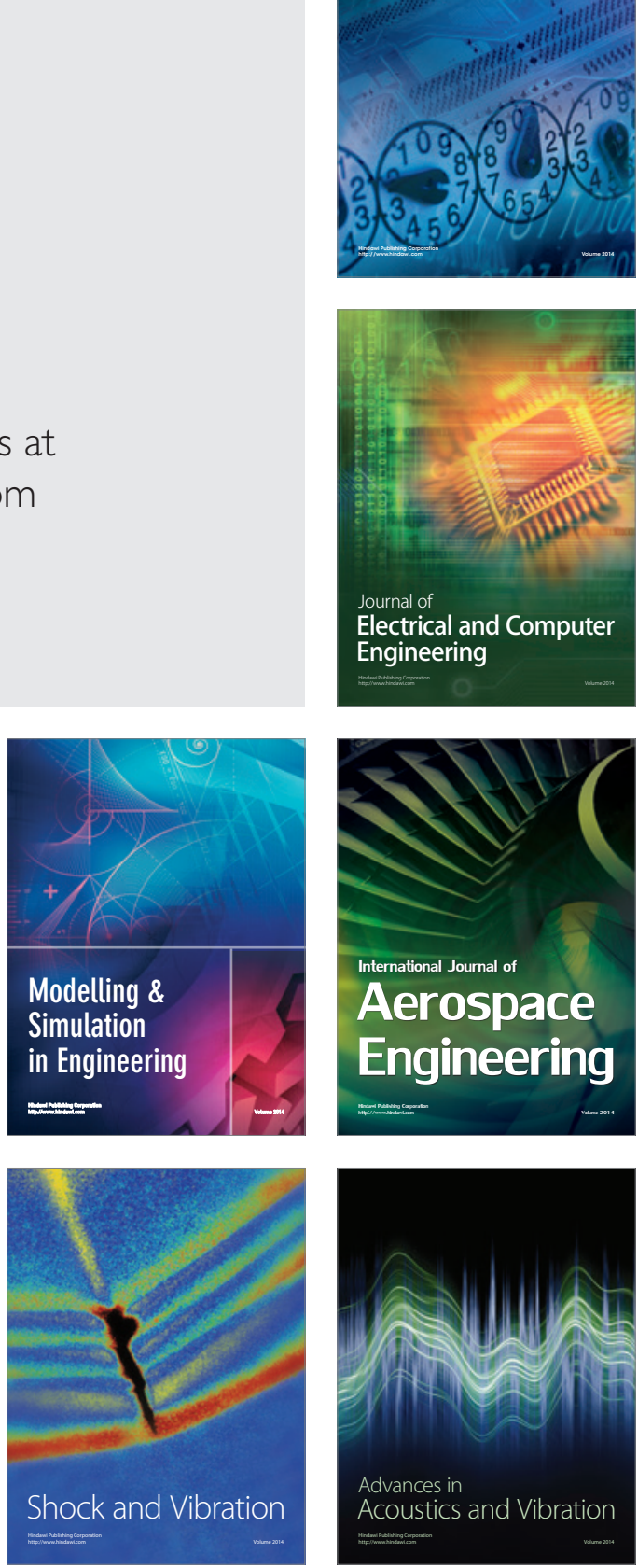